UNIVERSIDADE DE SÃO PAULO

FACULDADE DE MEDICINA DE RIBEIRÃO PRETO

ALEXIS GALENO MATOS

Influência do posicionamento relativo entre a fóvea e a cabeça do nervo óptico sobre o campo visual central de pacientes com glaucoma 


\title{
Influência do posicionamento relativo entre a fóvea e a cabeça do nervo óptico sobre o campo visual central de pacientes com glaucoma
}

\author{
Versão Corrigida \\ Versão original encontra-se na unidade que aloja o Programa de Pós- \\ Graduação
}

Tese apresentada à Faculdade de Medicina de Ribeirão Preto da Universidade de São Paulo para obtenção do Título de Doutor em Ciências.

Área de Concentração: Mecanismos Fisiopatológicos nos Sistemas Visual e AudioVestibular.

Orientador: Prof. Dr. Jayter Silva de Paula 
Autorizo a reprodução e divulgação total ou parcial deste trabalho, por qualquer meio convencional ou eletrônico, para fins de estudo e pesquisa, desde que citada a fonte.

Catalogação da Publicação

Preparada pela Biblioteca do Serviço de Biblioteca e Documentação

Faculdade de Medicina de Ribeirão Preto da Universidade de São Paulo

Matos, Alexis Galeno.

Influência do posicionamento relativo entre a fóvea e a cabeça do nervo óptico sobre o campo visual central de pacientes com glaucoma / Alexis Galeno Matos; orientador, Jayter Silva de Paula. - Ribeirão Preto, 2018.

76f.: 11 il. $30 \mathrm{~cm}$

Tese (Doutorado) - Faculdade de Medicina de Ribeirão Preto da Universidade de São Paulo. Departamento de Oftalmologia, Otorrinolaringologia e Cirurgia de Cabeça e Pescoço. Área de concentração: Mecanismos Fisiopatológicos nos Sistemas Visual e Áudio-Vestibular.

1. Glaucoma Primário de Ângulo Aberto. 2. Ângulo disco-fóvea. 3. Defeito de Campo Visual. 4. OCT.

CDD 999.99 


\section{FOLHA DE APROVAÇÃO}

\section{Aluno: Alexis Galeno Matos}

Título: Influência do posicionamento relativo entre a fóvea e a cabeça do nervo óptico sobre o campo visual central de pacientes com glaucoma

Tese apresentada à Faculdade de Medicina de Ribeirão Preto da Universidade de São Paulo para obtenção do Título de Doutor em

Ciências.

Área de Concentração: Mecanismos Fisiopatológicos nos Sistemas Visual e AudioVestibular.

Aprovado em:

Prof. Dr.

Instituição:

Assinatura:

Prof. Dr.

Instituição:

Assinatura:

Prof. Dr.

Instituição:

Assinatura:

Prof. Dr.

Instituição:

Assinatura:

Prof. Dr.

Instituição:

Assinatura: 


\section{Dedicatória}

Dedico primeiramente a Deus e toda minha família (pais, esposa e meu filho Levi), que me incentivaram e compreenderam minha ausência e as viagens para a realização deste estudo. Dedico, ainda, a todos os pesquisadores que direcionam sua vida no objetivo de melhorar a vida das outras pessoas através da ciência. 


\section{Agradecimentos}

Ao meu orientador Prof. Dr. Jayter Silva de Paula, pela atenção, paciência e pelo apoio durante todos os processos deste estudo, sendo um inspirador exemplo para meu aprimoramento científico, ético e profissional.

Ao Dr. Marcelo Jordão Lopes da Silva, que muito incentivou e contribuiu para o meu crescimento científico e intelectual.

À Faculdade de Medicina de Ribeirão Preto da Universidade de São Paulo, pela grande oportunidade de realização do Curso de Doutorado.

Ao Departamento de Oftalmologia, Otorrinolaringologia e Cirurgia de Cabeça e Pescoço da Faculdade de Medicina de Ribeirão Preto da Universidade de São Paulo. Em especial às funcionárias: Maria Cecília Onofre, por sua orientação, pelo apoio e processamento do material; e Eliane Santos Damasceno, pela paciência, pelo apoio, pela cordialidade e confiança.

Aos funcionários do Ambulatório de Oftalmologia do Hospital das Clínicas da Faculdade de Medicina de Ribeirão Preto da Universidade de São Paulo, em especial às técnicas: Lucélia Albiéri, Elisete M. G. da Silva e Maria Helena dos R. Almeida, pela cooperação, boa vontade e paciência durante a execução da pesquisa de campo deste estudo.

Aos colegas e amigos Tomas Teixeira Pinto e Pedro Fernandez Dalgalarrondo, pela cooperação e pelo auxílio na triagem e realização de exames junto aos participantes deste estudo.

Aos pacientes voluntários na realização deste projeto.

Ao espírito científico da eterna busca do conhecimento, do aprimoramento e do desenvolvimento humano.

À Coordenação de Aperfeiçoamento de Pessoal de Nivel Superior (CAPES), pelo auxílio financeiro concedido (Bolsa). 
Matos AG. Influência do posicionamento relativo entre a fóvea e a cabeça do nervo óptico sobre o campo visual central de pacientes com glaucoma. (Doutorado). Ribeirão Preto: Universidade de São Paulo, Faculdade de Medicina de Ribeirão Preto; 2018.

Objetivo: Investigar a influência do posicionamento da fóvea em relação à cabeça do nervo óptico (CNO) sobre resultados da perimetria padrão automatizada (PPA) central em pacientes com glaucoma e com defeitos patológicos localizados na rima temporal inferior (DTI) da CNO. Casuística e Métodos: Cinquenta e sete olhos de 35 pacientes com GPAA foram incluídos e divididos em dois grupos: um grupo com DTI (18 olhos) e outro com sem DTI (39 olhos). Três parâmetros diferentes obtidos a partir de uma tomografia de coerência óptica de domínio espectral (OCT-SD), ângulo do disco à fóvea (ADF), desvio vertical da fóvea (DVF) da linha média e diferença angular de defeito (DAD) entre borda DTI e ADF, foram correlacionados com quatro setores no programa 10-2: hemicampo superior, borda superior, borda nasal e arco súperonasal. As correlações foram testadas por meio de análises de regressão com modelos de efeitos mistos e interceptações aleatórias. Resultados: Os valores médios ( \pm DP [Desvio padrão]) de ADF, DAD e DVF foram, respectivamente: $-5,0$ \pm 4,4ํㅜ $43,3^{\circ} \pm 17,3^{\circ}$ e $-1346,6 \mu \mathrm{m} \pm 1609,0 \mu \mathrm{m}$. Comparações múltiplas mostraram que nos olhos com DTI, tanto DVF como DAD, mas não ADF, afetam significativamente os setores do campo visual (CV). As regressões binomiais de ROC demonstraram que apenas os valores de corte do 95 percentil de DVF ($3264,5 \mu \mathrm{m})$ e DAD $\left(70,5^{\circ}\right)$ podem promover mudanças de sensibilidade na borda superior dos setores do programa 10-2 [DVF: $A U C=0,60(95 \% \mathrm{Cl}=0,50-0,71)$; DAD: $A U C=0,83$ (IC 95\% = 0,70 - 0,912)]. Conclusões: A localização vertical da fóvea e o seu posicionamento relativo aos limites do DTI interferiram nas alterações de sensibilidade de pontos periféricos do hemicampo superior, no programa 10-2. Como o ADF, isoladamente, não influenciou significativamente nessas alterações, outros ajustes utilizando posicionamento foveal e parâmetro da OCT devem ser considerados na avaliação da PPA central em pacientes com defeitos glaucomatosos localizados na CNO.

Palavras-chave: Ângulo do disco-fóvea. Camada de fibras nervosas da retina. Campo visual central. Glaucoma. 
Abstract 
Matos AG. Influence of fovea and the optic nerve head positioning between central visual field in patients with glaucoma. (Thesis). Ribeirão Preto: Universidade de São Paulo, Faculdade de Medicina de Ribeirão Preto; 2018.

Purpose: To investigate the effects of the fóvea positioning relative to the optic disc on the 10-2 visual field (VF) in glaucoma patients with localized inferotemporal optic disc rim defects (ITD). Casuistic and Methods: Fifty-seven eyes of 35 POAG patients were included divided as having ITD (18 eyes) or not (39 eyes). Three different parameters obtained from a spectral domain optic coherence tomography (SD-OCT) [disc-fovea angle (DFA), fóvea vertical deviation (FVD) from midline, and defect's angular difference between ITD border and DFA (DAD)] were correlated to four 10-2 VF sectors: superior hemifield, superior edge, nasal edge, and supero nasal arch. Correlations were tested using regression analyses with mixed effects models and random intercepts. Results: Mean ( \pm SD) values of DFA, DAD, and FVD were respectively: $-5,0^{\circ} \pm 4.4^{\circ}, 43.30^{\circ} \pm 17.33^{\circ}$, and $-1346.6 \mu \mathrm{m} \pm 1609.0 \mu \mathrm{m}$. Multiple comparisons showed that in eyes with localized ITD both FVD and DAD, but not DFA, significantly affect the VF sectors. ROC binomial regressions demonstrated that only the 95th percentile cut-off values of FVD $(-3264,5 \mu \mathrm{m})$ and DAD $\left(70,5^{\circ}\right)$ may influence changes in one (superior edge sensitivity) of the 10-2 VF sectors [FVD: AUC $=0,60(95 \% \mathrm{Cl}=0,50-0,71)$; DAD: $A U C=0,83(95 \% \mathrm{Cl}=0,70-0,912)]$. Conclusions: The vertical fóvea location and its relative positioning to the limits of the ITD impact peripheral 10-2 VF points in the superior hemifield. As DFA itself did not show significant influences in the 10-2 VF, further adjustments using foveal positioning with OCT parameters should be considered in topographical analyses of the central VF in glaucoma patients with localized ITD.

Keywords: Disc-fovea angle. Retinal nerve fiber layer. Central visual field. Glaucoma 
Lista de Figuras 
Figura 1: Retinografia mostrando cabeça do nervo óptico com lesão glaucomatosa, caracterizada por aumento de sua escavação (dupla seta) e defeitos iniciais na camada de fibras nervosas da retina (cabeça de seta).

Figura 2: Exemplos de impressos da PPA, mostrando progressão da perda visual relacionada ao glaucoma, observadas com intervalos de quatro anos em paciente com controle inadequado da PIO.

Figura 3: Imagem representando o local da lesão (em vermelho) na CNO e CFNR em três situações distintas, gerando repercussão específica representada no campo visual de pacientes portadores de glaucoma.

Figura 4: Comparação da disposição dos pontos testados no programa 242 (vermelho) e no programa 10-2 (azul). Os pontos testados em comum estão pintados em verde. A área cinza delimita a região da mancha cega em uma PPA de olho direito.

Figura 5: Esquema demonstrando a delimitação dos setores propostos (áreas de sombra), programa 10-2, no hemicampo superior: BS - borda superior; HS - hemicampo superior; BN - borda nasal; SN - Arco súpero-nasal.

Figura 6: Adaptação da delimitação dos setores proposto por GarwayHeath (90), delimitando os quadrantes setoriais da distribuição da camada de fibras nervosas na CNO. T: temporal (311 $\left.-40^{\circ}\right)$, TS: temporal superior $\left(41^{\circ}-80^{\circ}\right)$, NS: nasal superior $\left(81^{\circ}-120^{\circ}\right)$,

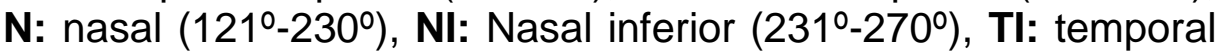
inferior $\left(271^{\circ}-310^{\circ}\right)$

Figura 7: llustração determinando os parâmetros usados na relação de posicionamento entre disco óptico e fóvea: 1 . Ângulo disco-fóvea (ADF); 2. Diferença angular do defeito (DAD); 3. Distância discofóvea; 4. Desvio vertical da fóvea (DVF)

Figura 8: Imagem demonstrando no gráfico TSNIT do OCT o local do inicio do defeito localizado. No caso aproximadamente 300․ DAD será (360 - 300) - ADF).

Figura 9: Imagem demonstrando exemplos de dois pacientes com ângulos disco-fóvea (ADF) comparáveis e defeitos localizados na borda temporal inferior apresentando diferentes padrões de perdas no setor de borda superior (BS) (delimitação cinza nos oito pontos superiores no desvio total) da PPA, programa 10-2. Note que o olho direito do segundo paciente apresenta pior perda de BS e 
desvio vertical da fóvea (DVF) grande e diferença angular de defeito (DAD) pequeno..

Figura 10: Imagem adaptada do modelo proposto por Hood et al. (2012) (92), estimando áreas de maior vulnerabiliade e sua influência na repercussão da lesão nas fibras maculares. A MVZ é a zona de vulnerabilidade macular na rima do disco óptico que se estende de $45^{\circ}$ a $69^{\circ}$

Figura 11: Imagem ilustrando variações da posição macular. Verificam-se os respectivos DAD, mostrando os ângulos 1, 2 e 3. O ângulo 3, por ter menor valor apresenta maior probabilidade de lesão na PPA central. (A, B e C) DVF das três posições. O DVF C, por ter maior valor apresenta maior probabilidade de lesão na PPA central. A correlação foi positiva no estudo em DAD menor que 70,5ํㅡㄹ $\mathrm{DVF}$ com valor maior $-3264,5 \mu \mathrm{m}$. 
Lista de Tabelas 
Tabela 1 - Tabela adaptada representando a expectativa do número de pessoas com glaucoma no mundo, em 2020, com base nos resultados apresentados por Quigley et al.....

Tabela 2 - Características demográficas e clínicas dos participantes, descritas pela presença de DTI ou ausência de defeitos localizados na borda do nervo

Tabela 3 - Resultados de correlação univariada entre fatores topográficos e perdas normalizadas em setores superiores selecionados da PPA, programa 10-2, em pacientes com DTI ou sem defeitos localizados . 49

Tabela 4 - Resultados significativos de comparações múltiplas dos fatores topográficos que influenciam as perdas normalizadas em setores superiores selecionados da PPA, programa 10-2, em pacientes com ou sem DTI 
Lista de Gráficos 
Gráfico 1 - Comparação do Ângulo Disco-Fóvea (ADF) e o número de olhos avaliados nos grupos DTI e controle, mostrando pouca disparidade entre os valores encontrados 
Lista de Símbolos e Abreviaturas 


$\begin{array}{ll}{ }_{-}^{-} & \text {Grau } \\ \boldsymbol{\mu m}- & \text { Micras } \\ \text { ADF - } & \text { Ângulo do disco-fóvea } \\ \text { AVCC- } & \text { Melhor acuidade visual corrigida }\end{array}$

BN- Borda nasal

BS- Borda superior

CFNR- $\quad$ Camada de fibras nervosas da retina

CGL- Corpo geniculado lateral

CGR- Células ganglionares da retina

CNO- Cabeça nervo óptico

CV- $\quad$ Campo visual

D- Dioptrias

DAD- Diferença angular do defeito

dB- Decibéis

DDF- Distância disco óptico à fóvea

DO- Disco óptico

DP- Desvio Padrão

DTI- Defeito Temporal inferior

DVF- Desvio vertical da fóvea

E/CNO- Escavação/Cabeça do Nervo Óptico

FDT- $\quad$ Frequency Doubling Technology

FoDi- $\quad$ Tecnologia de alinhamento Fóvea-Disco

GHT- $\quad$ Testes de glaucoma no hemicampo (do inglês, Glaucoma Hemifield Test)

GPAA- Glaucoma primário de ângulo aberto 
HCFMRP-USP- Hospital das Clínicas da Faculdade de Medicina de Ribeirão Preto da Universidade de São Paulo

HFA- $\quad$ Analisador de campo visual Humphrey (do inglês Humphrey Field Analyzer)

HS- $\quad$ Hemicampo superior

ISNT- Inferior-superior-nasal- temporal

logMAR- $\quad$ Escala linear logarítmica de perda da acuidade visual

MD- $\quad$ Desvio médio de sensibilidade (do inglês, Mean Deviation)

mmHg- Milímetros de mercúrio

MVZ- $\quad$ Zona de vulnerabilidade da mácula

OCT- Tomografia de coerência óptica

OCT-SD- $\quad$ Tomografia de coerência óptica de domínio espectral

OD- $\quad$ Olho direito

OE- $\quad$ Olho esquerdo

OMS- $\quad$ Organização Mundial de Saúde

p- $\quad$ Probabilidade

PIO- Pressão intraocular

PPA- $\quad$ Perimetria padrão automatizada

PSD- Desvio padrão (do inglês Pattern Standart Deviation)

SEN- Seno

SITA- Algoritmo de limiar interativo sueco (do inglês Swedish interactive threshold algorithm)

SN- $\quad$ Arco súpero-nasal

SWAP- Short Wavelength Automated Perimetry

TCLE- $\quad$ Termo de Consentimento Livre e Esclarecido

TD- $\quad$ Desvio total (do inglês, Total Deviation)

TSNIT- $\quad$ Temporal superior nasal inferior temporal 


\section{SUMÁRIO}

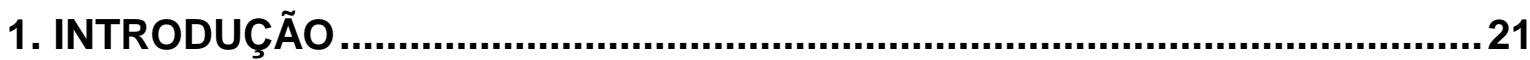

1.1. Glaucoma: definição, fatores de risco, classificação, etiologia .............................22

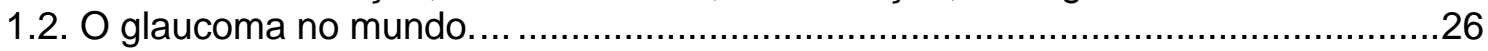

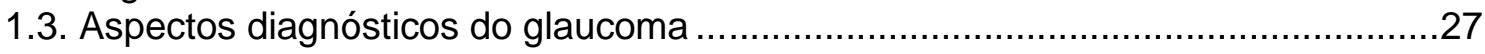

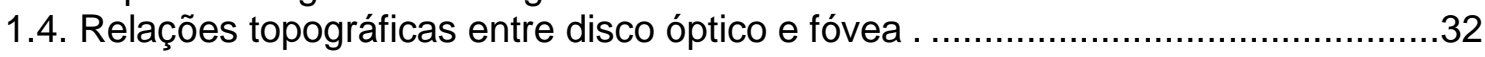

1.5. Justificativa

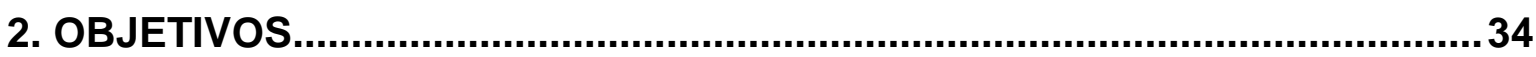

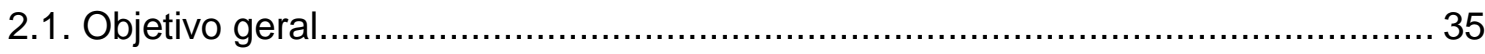

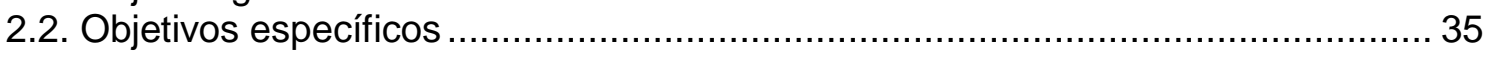

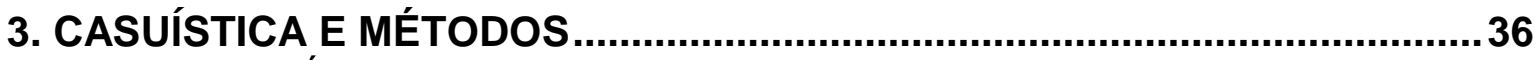

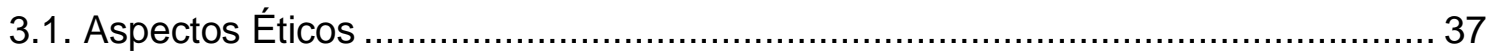

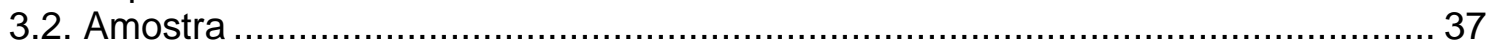

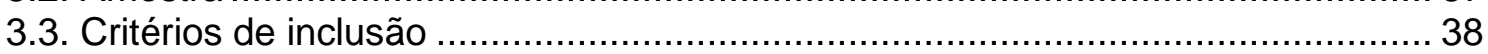

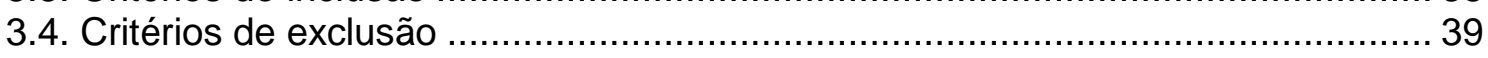

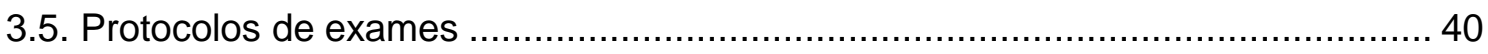

3.5.1. Perimetria visual computadorizada ................................................. 40

3.5.2. Divisão em setores da perimetria visual automatizada no programa 10-2.. 40

3.5.3. Tomografia de coerência óptica .............................................................41

3.5.4. Determinação do defeito localizado......................................................42

3.5.5. Relações topográficas entre o posicionamento da fóvea e do nervo

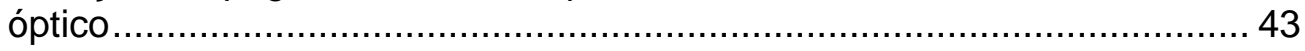

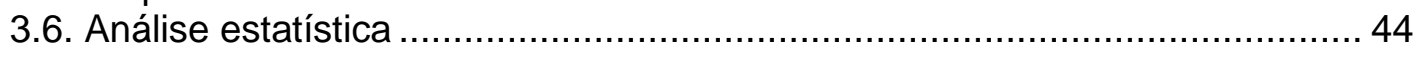

4. RESULTADOS

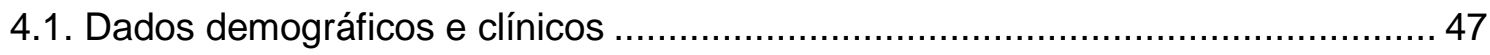

4.2. Correlação entre medidas da relação disco-fóvea com CV programa 10-2 ......... 48

5. DISCUSSÃO

6. CONCLUSÕES.................................................................................... 59

7. REFERÊNCIAS BIBLIOGRÁFICAS......................................................61

8. ANEXOS 


\section{1- Introdução}




\subsection{Glaucoma: definição, fatores de risco, classificação e etiologia}

A palavra glaucoma advém do grego "Glaukós", que significa brilhante, cintilante, cor azul-esverdeada ou a ideia de cor não determinada. Assim, segundo filósofos gregos, o glaucoma seria o resultado de um processo de "glaucosis" designando mudança da cor dos olhos, o que representaria um quadro patológico associado ao edema de córnea, atrofia da íris e até catarata, observado em casos de glaucoma agudo ou crônico $(1,2)$.

O glaucoma é uma neuropatia óptica progressiva, de etiologia multifatorial e complexa, que se apresenta em um conjunto heterogêneo de afecções com mecanismos patológicos distintos causando aumento da escavação da cabeça do nervo óptico (CNO) (Figura 1) associado à perda das células ganglionares da retina (CGR) e de seus axônios (3-7).

Figura 1: Retinografia mostrando cabeça do nervo óptico com lesão glaucomatosa, caracterizada por aumento de sua escavação (dupla seta) e defeitos iniciais na camada de fibras nervosas da retina (cabeça de seta)

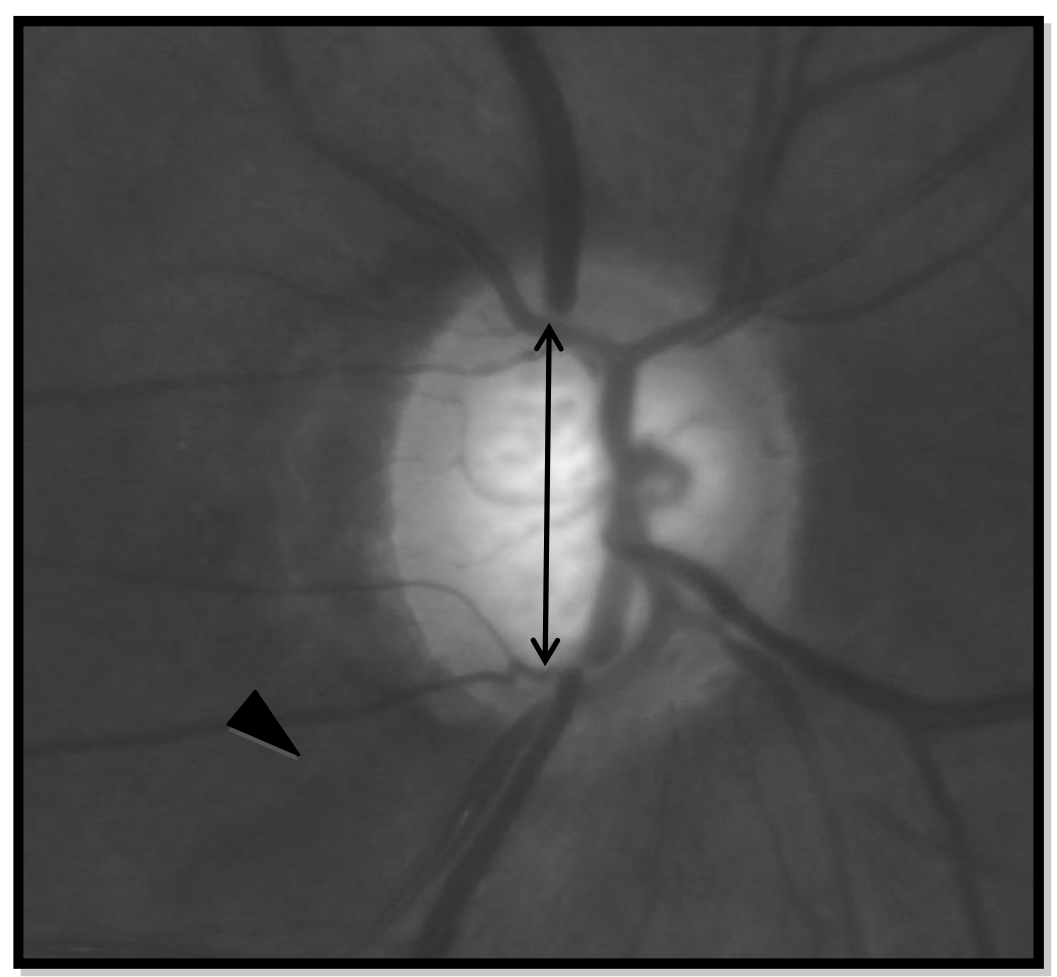


Os axônios das CGR têm distribuição que respeitam os limites da rafe mediana, sendo que aqueles oriundos da região macular nasal irão formar o feixe papilomacular, projetando-se diretamente na CNO. Os axônios localizados temporalmente à fóvea se direcionam aos polos superior e inferior da CNO seguindo um trajeto mais arqueado. A rafe mediana divide as fibras em superior e inferior obedecendo a uma linha imaginária entre a fóvea e a CNO nesta região (8). As fibras nervosas centrais seguem um trajeto mais superficial na camada de fibras nervosas da retina (CFNR), sendo que as originárias da retina periférica situam-se mais profundamente na região peripapilar (9). Mais de $90 \%$ da CFNR é composta por axônios das CGR que se projetam da retina para o corpo geniculado lateral (CGL), considerado o principal centro de conexão da visão no cérebro $(10,11)$.

O CGL apresenta dois tipos de neurônios: os interneurônios, que ficam confinados ao CGL, e os de transmissão, que se projetam para o córtex visual. Três populações de neurônios de transmissão são bem identificadas como os tipos $M$ (magnocelular), $P$ (parvocelular) e $K$ (koniocelular). As células $M$ são grandes e sensíveis ao contraste e ao movimento. As células $P$ são pequenas $e$ estão relacionadas à percepção de cores, principalmente ao espectro verdevermelho, mas com pouca sensibilidade ao contraste. Já as células $\mathrm{K}$ são as menores e estão envolvidas com a percepção azul-amarelo (12).

Os mecanismos fisiopatológicos descritos como responsáveis pela degeneração progressiva do nervo óptico; ou mais especificamente, pelas lesões das CGR que ocorrem no glaucoma, são relacionados a processo inflamatório crônico (13-15), processos isquêmicos (14) e apoptose (16-18). Inúmeros são os fatores associados a esses e outros processos relatados na literatura, cujo aprofundamento teórico foge do escopo desta tese.

A avaliação da CNO e CFNR é essencial para o reconhecimento das lesões glaucomatosas em qualquer fase da doença. A quantificação do diâmetro da escavação, proporcionalmente ao diâmetro da CNO, é um dos critérios usados na diferenciação de nervos normais e glaucomatosos. O aumento da relação dos diâmetros da escavação/cabeça do nervo óptico (E/CNO), assim como o aumento da E/CNO vertical, são indicadores da perda de rima neural, uma vez que os 
setores superior e inferior da CNO são considerados os de maior vulnerabilidade (19). Tais alterações da rima neural se acompanham, geralmente, de perdas na CFNR em regiões anatomicamente relacionadas, tanto para defeitos globais quanto para os localizados. Outros sinais, como a presença de hemorragias na CNO e atrofia da CFNR na região peripapilar, podem sugerir, ainda, início ou progressão da doença (20).

O glaucoma apresenta diversos fatores de risco conhecidos $(4,7,21)$, porém o mais significativo deles é o aumento da pressão intraocular (PIO), considerado, em muitos casos, como causa das lesões (4,22-24). O estágio da doença, a idade de início, o inadequado controle da PIO e a progressão da lesão durante o tratamento são fatores de risco para a cegueira no glaucoma (5,25-27). Assim, com a perda das CGR ocorrem alterações estruturais da CNO e, como consequência, progressão da doença correlacionada na perimetria padrão automatizada (PPA) (Figura 2).

Figura 2 - Exemplos de impressos da PPA, mostrando a progressão da perda visual relacionada ao glaucoma observada com intervalos de quatro anos em paciente com controle inadequado da PIO

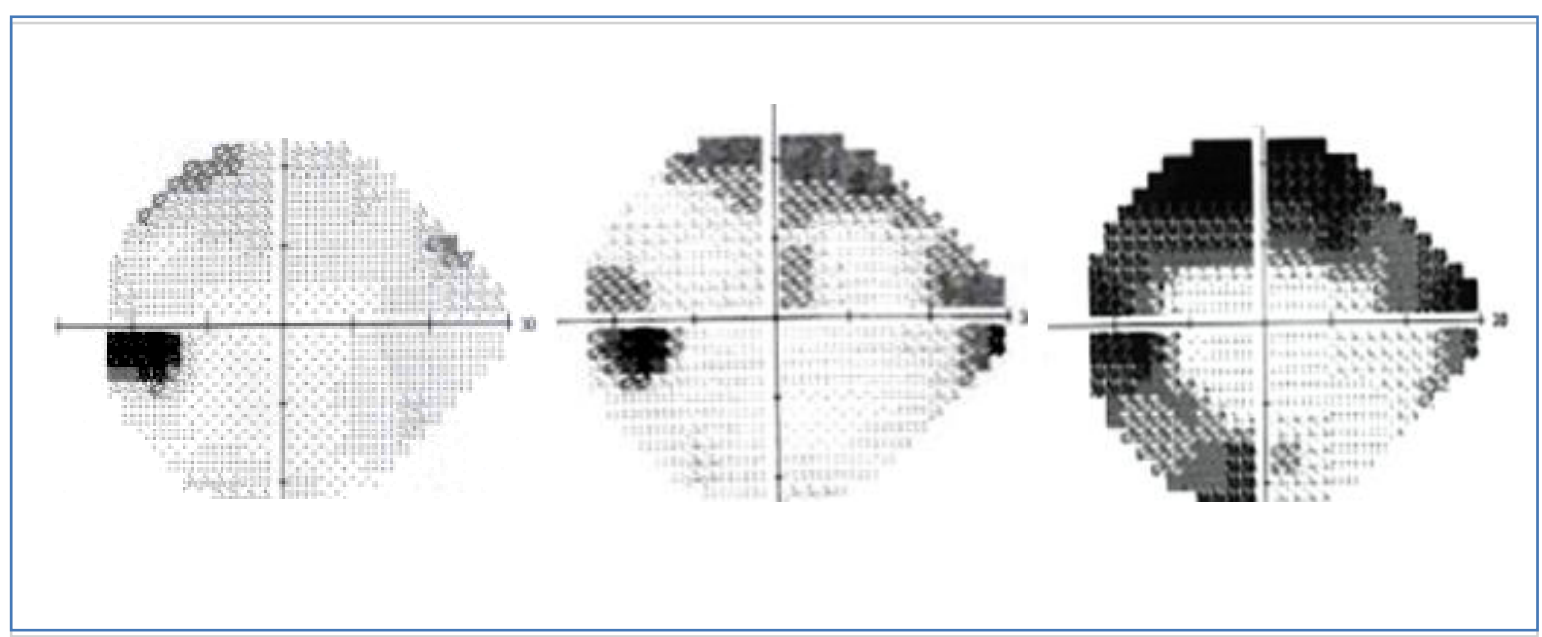


Desta forma, levando em consideração os principais estudos clínicos em glaucoma, destacam-se os seguintes fatores de risco para o glaucoma (levando em conta, primordialmente, o glaucoma primário de ângulo aberto - GPAA) (28):

- PIO - É o principal fator de risco, pois a ocorrência aumenta conforme os níveis e a flutuação da PIO aumentam; e a redução da PIO por meio de tratamento diminui o risco de progressão das lesões na PPA $(29,30)$.

- Idade - A frequência de glaucoma aumenta consideravelmente com o envelhecimento $(31,32)$, especialmente em pacientes caucasianos (33). No entanto, a prevalência da doença em indivíduos com mais de 75 anos ainda é maior na raça negra (34).

- História familiar - As taxas de risco variam nos diversos estudos, mas acredita-se que a chance possa dobrar ou ser até nove vezes maior quando há familiares com a doença $(35,36)$.

- Etnia - A prevalência é maior em pacientes de origem africana e latina do que em outros grupos, assim como a cegueira por glaucoma é cerca de seis vezes maior em negros $(29,31)$.

- Espessura central da córnea - A presença de córneas com espessura central menor é considerada um fator de risco associado ao glaucoma. Ainda não existem normograma ou tabela de correção da espessura e valor real da PIO completamente validadas (uma vez que a PIO é subestimada nas leituras da tonometria de aplanação em córneas mais finas) $(29,37)$.

- Pressão de perfusão ocular - A pressão de perfusão ocular pode estar relacionada às alterações na irrigação, observadas na CNO no GPAA, tomando-se sua definição de diferença entre a pressão arterial (sistólica, diastólica ou média) e a PIO $(36,38)$.

As formas mais usuais de se classificar o glaucoma são baseadas na etiologia e no seu mecanismo fisiopatológico, devendo-se levar em conta as relações anatômicas da câmara anterior que podem levar ao aumento da PIO. Considerando a gama de propostas de classificações existentes na literatura, os 
detalhes descritos nesta seção foram compilados, principalmente dos estudos de Foster et al. (39) e Wolfs et al. $(39,40)$.

Deve-se ressaltar que a determinação da causa inicial da doença deve ser buscada, uma vez que permite a classificação etiológica e direciona o tratamento específico juntamente com a redução da PIO (41). Assim, para a classificação com base etiológica considera-se o conhecimento ou não de um fator causal, seguindo a denominação de "primário" - quando desconhecido - e "secundário" quando conhecido (39).

$\mathrm{Na}$ classificação "primária", estariam o GPAA, o glaucoma de pressão normal (considerado por muitos como uma variante do GPAA), o glaucoma primário de ângulo fechado, glaucoma congênito primário e os glaucomas ocasionados por mecanismos combinados. Dentre as causas dos glaucomas "secundários", podem ser incluídas as doenças relacionadas a alterações do endotélio corneal, corpo ciliar e íris, cristalino e da retina e do vítreo, tumores intraoculares, elevação da pressão venosa episcleral, inflamação ocular, uso de corticoides, traumas e cirurgias, dentre outras $(39,42)$.

A classificação voltada aos mecanismos fisiopatológicos envolvidos na gênese da doença distingue os glaucomas de acordo com as condições do sistema de drenagem do humor aquoso, a saber: a) ângulo iridocorneal aberto (dividido em alterações pré-trabeculares, trabeculares e pós-trabeculares), b) fechado (dividido em mecanismos de adesão ou aposição da periferia da íris com ou sem bloqueio pupilar) e c) com anomalias de desenvolvimento $(1,28)$.

\subsection{0 glaucoma no mundo}

O glaucoma é uma das principais causas de deficiência visual no mundo (43). Relatos recentes estimaram 60,5 milhões de pessoas com diagnóstico de glaucoma para o ano de 2010 e 79,6 milhões para o ano de 2020, resultando em 8,4 e 11,2 milhões de indivíduos com cegueira bilateral, respectivamente $(3,44)$.

Embora dados mundiais, incluindo aqueles da Organização Mundial da Saúde (OMS), atribuírem ao glaucoma 12,3\% dos casos de cegueira em 2002 (44-46), revisões recentes descrevem a proporção de aproximadamente 8\% (46) 
e 6,6\% (47) desses casos em 2010. Ainda assim, esta grave afecção continua no posto de segunda maior causa de cegueira no mundo e no de primeira causa irreversível (Tabela 1) $(3,45,46)$.

O glaucoma é, ainda, responsável por um impacto social, médico e financeiro cada vez mais significativo. Sua prevalência varia segundo localização, raça, idade e características socioeconômicas da população estudada $(48,49)$. Alguns autores sugeriram que as probabilidades de desenvolvimento de cegueira unilateral e bilateral devido ao GPAA no decorrer de 15 anos seriam de $27 \%$ e $9 \%$, respectivamente (50). O risco de perda de visão no GPAA parece ser ainda maior nos indivíduos da raça negra, na faixa etária acima de 60 anos, e nos países em desenvolvimento $(50,51)$.

Tabela 1 - Tabela adaptada representando a expectativa do número de pessoas com glaucoma no mundo, em 2020, com base nos resultados apresentados por Quigley et al. (3)

\begin{tabular}{lcc}
\hline Região mundial & Portadores de Glaucoma & Porcentagem da população >40 anos \\
\hline China & $21,824,015$ & $3,05 \%$ \\
Índia & $16,088,243$ & $2,64 \%$ \\
Europa & $13,971,113$ & $2,40 \%$ \\
África & $8,359,451$ & $4,39 \%$ \\
América Latina & $8,011,575$ & $3,60 \%$ \\
Japão & $3,084,669$ & $3,96 \%$ \\
Mundo & $79,640,184$ & $2,86 \%$ \\
\hline
\end{tabular}

Fonte: Adaptado de Quigley et al, 2006 (3).

\subsection{Aspectos diagnósticos do glaucoma}

Uma vez que na investigação clínica do glaucoma não há ainda um exame único que permita o diagnóstico inicial da doença em todos os casos, se preconiza a associação de resultados de exames complementares. A despeito da importância da medida da PIO e avaliação de diversos componentes do segmento 
anterior (tonometria, biomicroscopia, paquimetria e gonioscopia) para 0 diagnóstico e classificação da doença, exames voltados para avaliação estrutural da retina e do nervo óptico e avaliação funcional relacionada são indispensáveis nessa propedêutica (28).

O registro estrutural e a análise da CNO e da CFNR podem ser realizados por meio de estereofotografia colorida da retina voltada ao nervo óptico, com atenção aos aspectos da escavação do nervo óptico e da camada de fibras circunjacentes; considerada, ainda, padrão-ouro para o glaucoma. Atualmente, essa análise de fibras é realizada por pela: tomografia de coerência óptica (OCT) $(4,28)$. Existem outras propostas de avaliação complementar no glaucoma, mas com limitações para uso rotineiro, como os exames voltados ao estudo do contraste e os eletrorretinográficos (eletrorretinogramas e potencial visual evocado) $(35,39)$.

O OCT permite, de forma não invasiva, a obtenção de imagens de alta resolução que quantificam a espessura da CFNR na região peripapilar e macular, além de realizar avaliação de parâmetros da CNO por meio de atraso e intensidade de luz retrodifundida das camadas retinianas usando uma técnica de correlação óptica conhecida como interferometria. Atualmente, a tecnologia de domínio espectral do OCT (OCT-SD) aumentou a velocidade e sensibilidade das análises realizadas, sendo capaz de detectar mais precocemente danos estruturais, muitas vezes prévios às alterações funcionais da PPA $(52,53)$.

A avaliação funcional do campo visual (CV) baseada na PPA é de longa data uma das mais importantes no diagnóstico e seguimento do glaucoma. Os defeitos no CV no glaucoma são, em geral, correspondentes aos defeitos frequentemente observados nos quadrantes superiores e inferiores da CNO e às lesões da CFNR. As perdas iniciais na PPA são, geralmente, descritas como perdas periféricas pouco específicas, mas estudos mostraram que em alguns pacientes com glaucoma as lesões iniciais foram identificadas na região central da PPA (54-56). Defeitos focais, descritos quase concomitantemente, constituem a evidência mais clara e definitiva de perda visual por glaucoma $(57,58)$. 
As perdas de CV em pacientes com glaucoma são explicadas, em última análise, pelo dano às CGR e podem ser detectadas pela trajetória anatômica dos axônios na CFNR (Figura 3) (59,60).

Figura 3 - Imagem representando o local da lesão (em vermelho) na CNO e na CFNR em três situações distintas gerando repercussões específicas representadas no campo visual dos pacientes portadores de glaucoma.

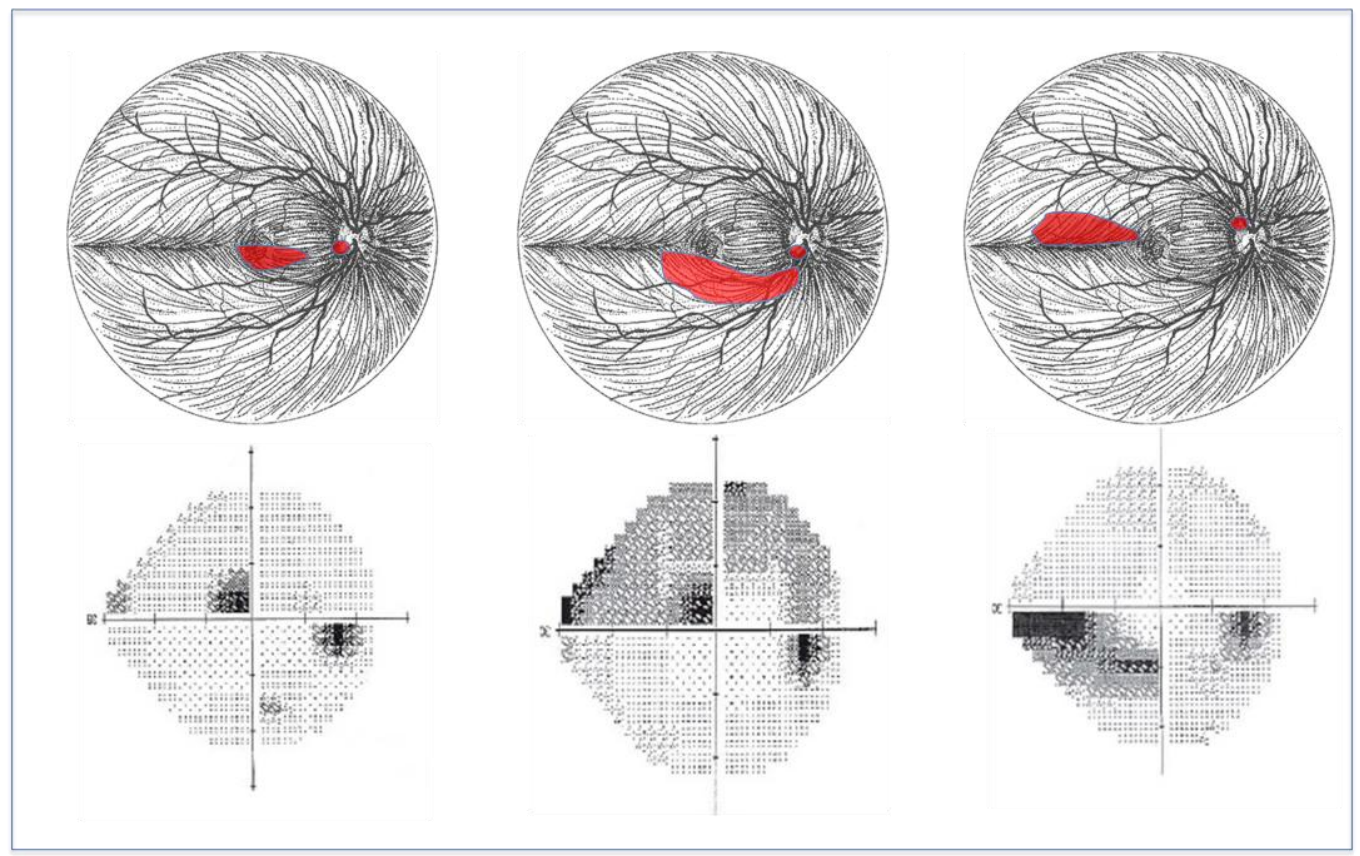

As lesões paracentrais estão envolvidas quando as áreas próximas à inserção do feixe papilomacular na CNO são afetadas. Essas lesões causam danos às atividades diárias das pessoas e podem ocorrer tanto em glaucomas avançados quanto em estágios iniciais da doença $(61,62)$. Alguns estudos relataram que mais de $50 \%$ dos olhos com glaucoma leve a moderado apresentaram defeitos nos três graus centrais (56), considerados importantes no desempenho visual dos pacientes.

Os exames de avaliação do CV são realizados, preferencialmente, com perímetros automatizados que usam estímulos estáticos brancos em fundo branco, tais como o Humphrey Field Analyzer (HFA, Carl Zeiss Meditec, Dublin, 
CA) e o Octopus (Haag-Streit AG, Koeniz-Berne, Switzerland). Ambos os aparelhos são os mais difundidos comercialmente e apresentam acurácia semelhante em termos de diagnóstico de lesões glaucomatosas (63). Outras tecnologias foram propostas no passado, como por exemplo, o Short Wavelength Automated Perimetry (SWAP, Carl Zeiss Meditec, Inc., Dublin, CA) e o Frequency Doubling Technology (FDT, Carl Zeiss Meditec, Inc., Dublin, CA), que buscam a identificação precoce da doença por meio de estimulação seletiva de diferentes tipos de CGR. No entanto, sua capacidade diagnóstica e aplicação clínica se mostraram limitadas $(64,65)$.

Os programas apresentados pelo HFA têm sido os mais estudados e usados no diagnóstico e acompanhamento dos pacientes com glaucoma. Por meio de pesquisa no "Pubmed" (www.pubmed.com), em 19 de março de 2018, observou-se que este aparelho foi o que apresentou maior número de estudos relacionados à doença, publicados naquela base de dados (HFA: 1194 artigos versus Octopus: 326 artigos). O protocolo padrão mais usado é o programa 24-2, que testa 54 pontos separados por seis graus nos 24 graus centrais de visão do paciente, com extensão até $030^{\circ}$ graus no quadrante nasal. Assim, apenas quatro pontos estão presentes dentro dos oito graus centrais em que se encontram aproximadamente $30-50 \%$ das células ganglionares da retina. Além disso, esses quatro pontos não representam perfeitamente a região de maior densidade de CGR, demonstrada pelo exame de OCT, quando o deslocamento das CGR na fóvea é considerado $(55,66)$.

O programa 10-2 do HFA busca detectar defeitos localizados nos 10 graus centrais, testando 68 pontos separados por dois graus entre eles, sendo mais específico para estudo de lesões de campo, mais restritas à região macular (Figura 4) (67). 
Figura 4 - Comparação da disposição dos pontos testados no programa 24-2 (vermelho) e no programa 10-2 (azul). Os pontos testados em comum estão pintados em verde. A área cinza delimita a região da mancha cega em uma PPA de olho direito.

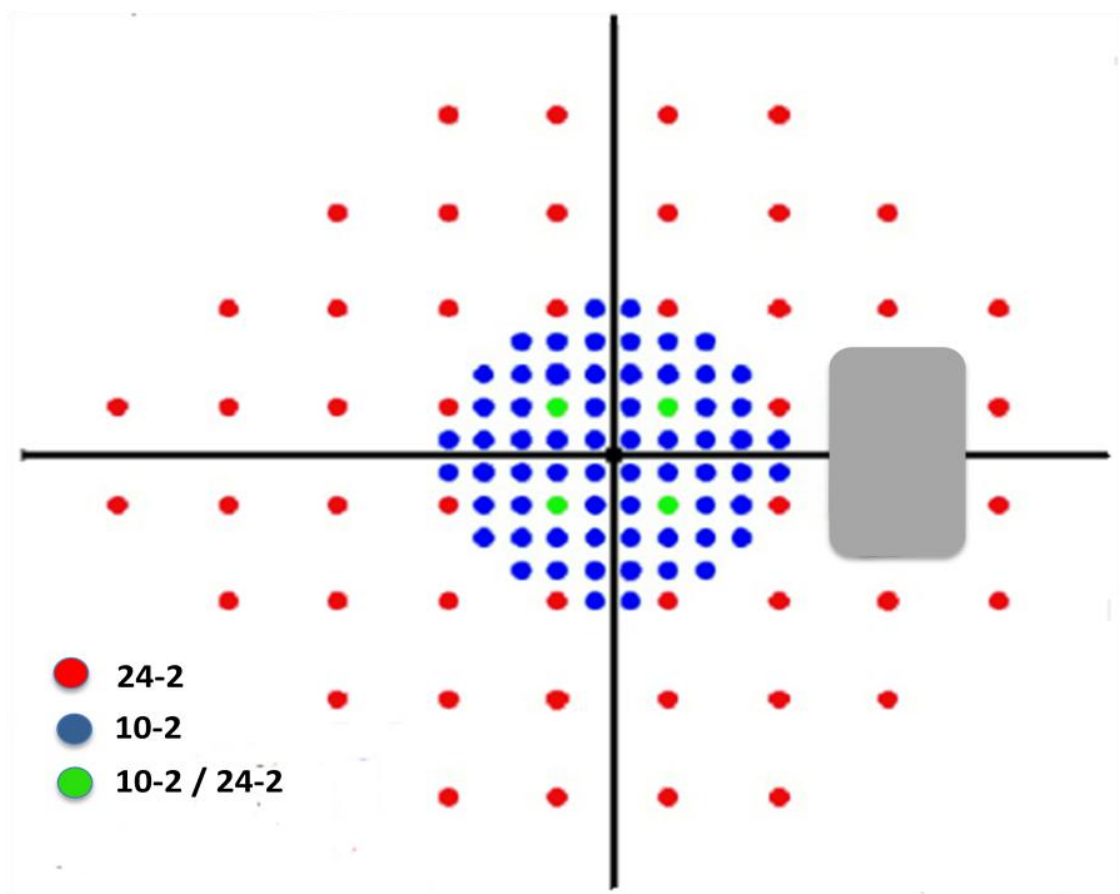

Recentemente, este programa adquiriu novamente importância, após alguns estudos mostrarem que o diagnóstico de progressão dos defeitos no CV são melhor observados em testes com menores intervalos de distância entre os pontos testados $(56,68,69)$. Traynis et al. $(55)$ relataram a existência de alterações de PPA no programa 10-2 em pacientes com glaucoma que se apresentaram normais no programa de análise 24-2.

Em um estudo projetado explicitamente para avaliar os defeitos iniciais, Heijl e Lundqvist (54) avaliaram 45 olhos que progrediram de PPA normal para anormal. Embora o maior número de pontos na PPA, inicialmente anormais, tenha ocorrido a $15^{\circ}$ da fixação, houve uma série de pontos anormais a $5^{\circ}$, especialmente nasais e na PPA superior. Mais recentemente, Schiefer et al. (70) relataram que mais de $50 \%$ dos olhos com glaucoma leve a moderado apresentavam defeitos dentro da central $\pm 3^{\circ}$. 


\subsection{Relações topográficas entre disco óptico e fóvea}

A CNO está localizada, usualmente, numa posição nasal superior em relação à fóvea. Para facilitar a correlação com textos publicados na língua inglesa e o entendimento de variáveis apresentadas neste estudo, a CNO será mencionada nas relações topográficas analisadas como o disco óptico (DO), ou apenas "disco". Assim, o ângulo disco-fóvea (ADF) existente varia em média de $-4,5^{\circ}$ a $-7,0^{\circ}$, sendo que ângulos negativos representam o posicionamento da fóvea abaixo da linha média horizontal (71-73).

A distância entre o disco e a fóvea (DDF), juntamente com o ADF, foram pouco estudados até o momento e possuem grande valor na avaliação funcional do nervo óptico e da CFNR no polo posterior do olho $(74,75)$. Além das relações anatômicas, ambas as medidas podem também estar relacionadas a alterações na acuidade visual e CV do paciente, principalmente naqueles com maiores DDF (76).

A investigação da área macular surgiu após a evolução substancial nos dispositivos de análise da espessura da retina com o OCT-SD, que apresenta maior resolução, atualmente indispensável para medições refinadas de diversas estruturas e também para determinar e corrigir o posicionamento relativo entre a fóvea e a CNO. O posicionamento da região foveal e a combinação das distâncias geradas com isso podem afetar a distribuição da CFNR macular (73), influenciando na apresentação estrutural e funcional dos defeitos glaucomatosos $(36,69,77)$.

Em estudos recentes, o glaucoma tem sido associado a lesões no complexo CGR macular, com consequências para a espessura da camada de fibras nervosas desta região (8,78-80). Ainda, uma vez que os feixes de CFNR na parte inferior do quadrante temporal foram considerados de maior vulnerabilidade, diferentes posições relativas entre a fóvea e o CNO podem acarretar maior risco de lesões nessa localização.

Considerando que a fóvea está, geralmente, localizada abaixo da linha horizontal mediana, em posição temporal inferior à CNO, e que a distribuição da CFNR é assimétrica geralmente, alguns autores sugerem maior fragilidade da 
CFNR na região inferior aos danos glaucomatosos (78). Logo, olhos com ADF mais negativos seriam mais suscetíveis aos danos e apresentariam fibras temporais superiores projetadas mais próximas à linha média - fatos que levariam a artefatos na PPA relacionados a diferenças anatômicas individuais $(81,82)$.

A PPA central é considerada como a mais apropriada para se investigarem defeitos de CGR maculares, apresenta-se centralizada na fóvea (fixação central) e com orientação que não acompanha obrigatoriamente o ADF. Assim, defeitos localizados na CFNR ou rima neural temporal inferior podem produzir perdas de sensibilidade variáveis no hemicampo superior (HS) em testes do programa 10-2 do HFA (83).

\subsection{Justificativa}

A variabilidade anatômica da disposição das estruturas do polo posterior do olho, incluindo o ADF, não está ainda completamente elucidada e pode se correlacionar com diferenças nos achados de exames complementares que se baseiam direta ou indiretamente na distribuição das CFNR e na arquitetura da rima neural da CNO.

Considerando tais variações anatômicas, justifica-se 0 estudo da correlação do posicionamento relativo entre a fóvea e o DO com possíveis alterações funcionais relacionadas ao glaucoma. Com isso, será possível reconhecer com maior precisão o impacto desta relação topográfica sobre os exames de PPA central, de forma a propor estratégias de correspondência e alinhamento espacial mais precisas e individualizadas, permitindo ainda eventuais modificações futuras nos critérios de diagnóstico e seguimento dos pacientes. Para tanto, foram investigados os efeitos do desvio vertical da fóvea (DVF) em relação à linha média horizontal sobre achados perimétricos usando o programa 10-2 em pacientes com defeito temporal inferior (DTI) de CNO glaucomatosos. 
2. Objetivos 


\subsection{Objetivo geral}

Analisar a influência da variabilidade anatômica do posicionamento relativo entre a CNO e a fóvea sobre alterações perimétricas de pacientes com GPAA.

\subsection{Objetivos específicos}

- Correlacionar os valores do ADF, obtidos com o OCT, com alterações de sensibilidade no HS da PPA central, programa HFA 10-2, em pacientes com GPAA e DTI.

- Verificar a influência do DVF em relação à linha horizontal mediana e da distância angular entre o DTI e a fóvea sobre as alterações de sensibilidade da PPA central, descritas acima, para os mesmos pacientes. 
3. Casuistica e Métodos 


\subsection{Aspectos Éticos}

O presente estudo foi aprovado pelo Comitê de Ética em Pesquisa do Hospital das Clínicas da Faculdade de Medicina de Ribeirão Preto da Universidade de São Paulo (HCFMRP-USP) Processo no 1.887.298 (Anexo A).

Todos os participantes submetidos aos procedimentos de pesquisa assinaram o Termo de Consentimento Livre e Esclarecido (TCLE) (Anexo B), após receberem detalhadas explanações sobre todos os procedimentos relacionados, seus potenciais efeitos benéficos e suas complicações, além de seu caráter voluntário. Este estudo não incorreu em qualquer prejuízo, lesões ou desconforto aos participantes e serviu para formação de banco de dados clínicos de pacientes com glaucoma neste hospital.

\subsection{Amostra}

Foi realizado estudo transversal utilizando informações de um banco de dados formado por pacientes atendidos previamente no Setor de glaucoma do HCFMRP-USP e do banco de dados de pacientes atendidos pelo Departamento de oftalmologia da Columbia University Medical Center, Nova York, EUA, previamente aprovado pelos instrumentos regulatórios locais.

Os pacientes foram divididos em dois grupos. O primeiro grupo foi composto por pacientes com GPAA (variantes de PIO alta ou normal) com DTI determinado por concordância, seguindo os critérios descritos no subitem 3.5.4. O segundo grupo, controle, foi formado por pacientes com GPAA (variantes de PIO alta ou normal), que diferiam do primeiro grupo somente por não apresentarem DTI da rima neural da CNO ou na CFNR. 


\subsection{Critérios de inclusão}

Foram incluídos pacientes de ambos os sexos, com idade entre 20 e 80 anos, que apresentavam os seguintes achados:

\section{Para ambos os grupos:}

Diagnóstico de GPAA descrito em prontuário médico, com exame oftalmológico demonstrando em pelo menos um olho:

- Ângulo gonioscópico classificado como aberto, sem alterações patológicas visíveis.

- Relação E/CNO igual ou superior a 0,6, avaliada pela biomicroscopia de fundo em lâmpada de fenda, evidenciando pelo menos um dos sinais relacionados ao glaucoma na CNO (assimetria entre os olhos da relação E/CNO maior que 0.2 , hemorragia de disco, alteração da sequência inferior-superior-nasal- temporal (ISNT), atrofia peripapilar, fosseta adquirida de nervo óptico, vasos em passarela, baioneta, anasalados ou colaterais, aumento das estrias da lâmina crivosa e escavação nasal);

- Defeitos sugestivos de glaucoma na PPA, segundo os critérios de Hodapp-Parrish-Anderson e Schimiti (84-86): Mean Deviation (MD) melhor do que -12 dB no programa 24-2 Swedish Interactive Thresholding Algorithm (SITA) - Standard do HFA com a adequada correção óptica, apresentando ao menos uma entre as três condições: três ou mais pontos adjacentes com $p<5 \%$ (no gráfico total deviation [TD]), sendo pelo menos um ponto com $\mathrm{p}<1 \%$; Glaucoma Hemifield Test (GHT) com descrição outside normal limits ou borderline; índice Pattern standard deviation (PSD) com nível menor que 5\% $(84,87,88)$. Apenas os exames com dados confiáveis permaneceram no estudo, sendo considerados exames não confiáveis aqueles com evidência de perdas de fixação de $>20 \%$ ou taxas de resposta falso-positivas $>15 \%$ e negativas $>20 \%$. 
- Eixo visual livre, sem opacidades que impedissem os exames oftalmológicos;

- Ausência de qualquer comorbidade ocular que potencialmente interferisse nas avaliações ou na função visual, como por exemplo, retinopatias, catarata, opacidades de córnea, alterações de anexos oculares, afecções neuro-oftalmológicas, complicações de cirurgia de catarata, tumores e inflamações intraoculares, dentre outros.

- Pacientes com refração esférica igual ou inferior a \pm 6 D (dioptria) e cilíndrica, igual ou inferior a $3 \mathrm{D}$;

- Ausência de outras cirurgias oftalmológicas prévias, à exceção de trabeculectomia e facectomia, se realizadas há mais de seis meses;

- Pacientes com a acuidade visual corrigida (AVCC) em ambos os olhos, igual ou melhor que 0,2 logMAR;

\section{Para o grupo DTI:}

Adicionalmente ao descrito previamente, dois especialistas em glaucoma avaliaram os exames dos pacientes a fim de se distinguirem os olhos que apresentavam DTI, usando os critérios citados no subitem 3.5.4.

\subsection{Critérios de exclusão}

- Paciente com incapacidade cognitiva ou de participação no estudo;

- Pacientes cujos um exame ou mais exames não obtiveram resultados confiáveis;

- Pacientes que não realizaram todos os exames do protocolo de estudo;

- Comorbidades sistêmicas em estado grave ou mal controladas. 


\subsection{Protocolo de exames}

Todos os participantes selecionados do banco de dados haviam sido submetidos à sequência de exames: oftalmológico clínico completo, PPA central e OCT-SD. O exame oftalmológico clínico incluiu exames de AVCC, motilidade ocular, refração, biomicroscopia, tonometria e gonioscopia. Foram descartados os pacientes do banco de dados que não se realizaram todos os exames.

\subsubsection{Perimetria visual computadorizada}

Todos os pacientes foram submetidos ao exame de PPA central (HFA 750, Carl Zeiss, Dublin, California), no programa 10-2 (estratégia SITA-STANDARD), com a adequada correção óptica para perto, pelo mesmo profissional técnico. Para fins de avaliação, foram consideradas alterações do gráfico TD, sendo este exame tomado como padrão para o indivíduo. Foram usados os critérios de confiabilidade, citados anteriormente, e os exames classificados como "baixa confiabilidade" foram excluídos da análise.

\subsubsection{Divisão em setores da perimetria visual automatizada no programa 10-2}

Com o objetivo de avaliar os pontos capazes de detectar redução da sensibilidade relacionada às perdas de CGR determinadas pelo DTI, foram utilizados apenas os achados superiores de HS de PPA central confiáveis. Além disso, foram determinados arbitrariamente três setores da PPA central no programa 10-2: a) a borda superior (BS - delimitada pelos oito pontos superiores); b) a borda nasal (BN - delimitada pelos quatro pontos nasais adjacentes à linha média horizontal); c) o arco súpero-nasal (SN - determinado por todos os pontos BS e BN mais o ponto na localização n5s5) (Figura 5). A média dos valores de sensibilidade e o número de pontos significativamente alterados $(p<0,1 \%)$ dessas áreas (HS, BS, BN e SN) foram considerados para análise estatística. 
Figura 5 - Esquema demonstrando a delimitação dos setores propostos (áreas de sombra) no programa 10-2 hemicampo superior - BS - borda superior; HS - hemicampo superior; BN - borda nasal; SN - arco súpero-nasal.

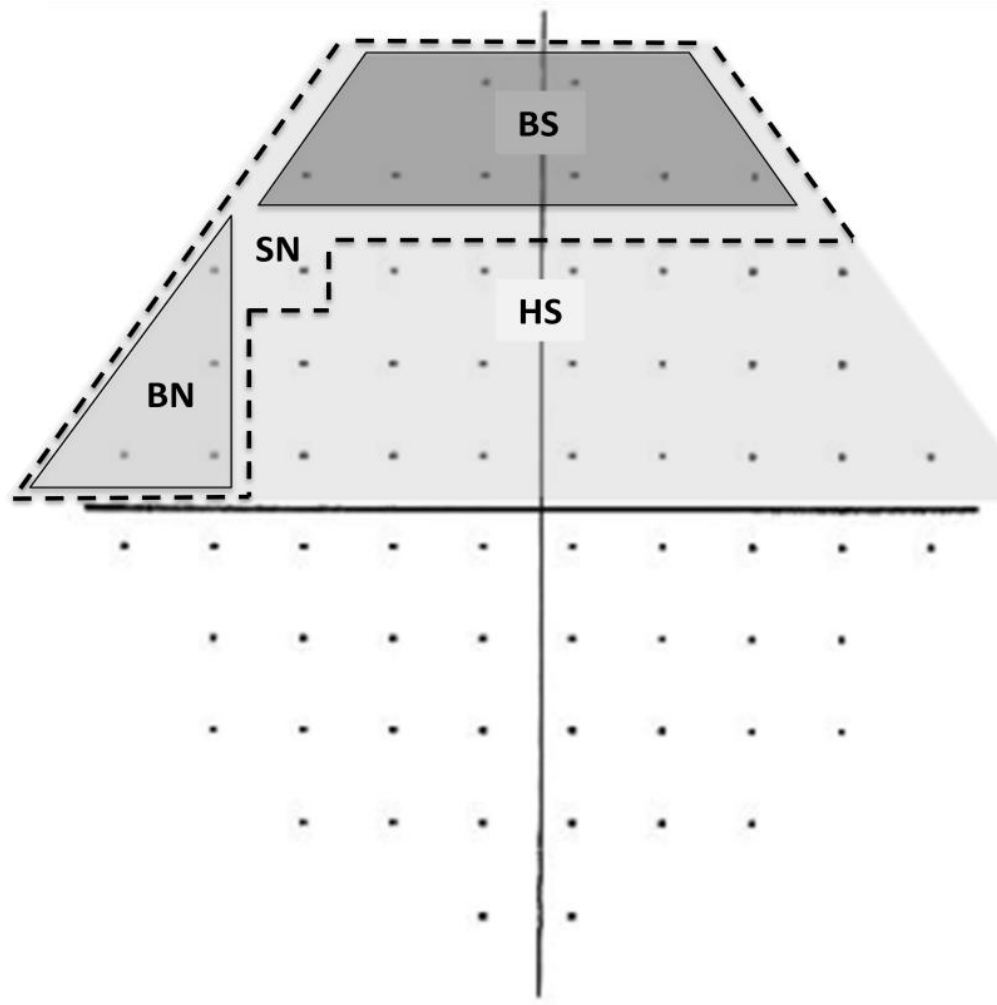

\subsubsection{Tomografia de coerência óptica}

Foram realizados exames de avaliação da estrutura retiniana e da CNO por meio de OCT-SD (Spectralis; Heidelberg Engeneering, Alemanha). Com essa metodologia foi possível documentar com precisão alterações de toda a extensão da retina, assim como medir a espessura da camada de fibras nervosas, por meio de protocolos de análise da CFNR (RNFL Thickness) próximo à CNO (global, por quadrantes e assimetria), da espessura macular (horizontal no feixe papilomacular e mapa macular) e do estudo de varredura de áreas de interesse da retina e nervo óptico, buscando documentar as características e extensão do DTI.

Por meio do sistema de tecnologia de alinhamento do disco à fóvea (FoDi) foi determinado o grau angular existente entre a CNO e a região macular (ADF). 
Apenas exames com excelente qualidade, maior que $20 \mathrm{~dB}$, sem sinais de descentração ou artefatos, foram utilizados na análise do estudo.

\subsubsection{Determinação do defeito localizado}

O DTI foi caracterizado por um defeito localizado na rima do nervo óptico visualizado nos exames de fundoscopia e confirmado pela análise da CFNR realizada pelo OCT. Dois especialistas em glaucoma avaliaram os exames dos participantes e categorizaram todos os olhos como apresentando um DTI na borda da CNO e CFNR usando o diagrama de Garway-Heath (89) (Figura 6), confirmado com perda significativa no mesmo setor por OCT-SD, utilizando a estratégia setorial de espessura da CFNR.

Figura 6 - Adaptação da delimitação dos setores proposta por Garway-Heath (89), delimitando os quadrantes setoriais da distribuição da camada de fibras nervosas na CNO. T: temporal $\left(311^{\circ}-40^{\circ}\right)$, TS: temporal superior $\left(41^{\circ}-80^{\circ}\right)$,

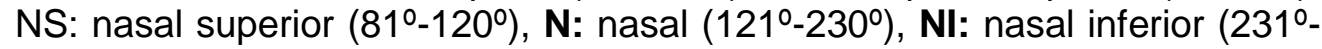
$\left.270^{\circ}\right)$ e TI: temporal inferior $\left(271^{\circ}-310^{\circ}\right)$

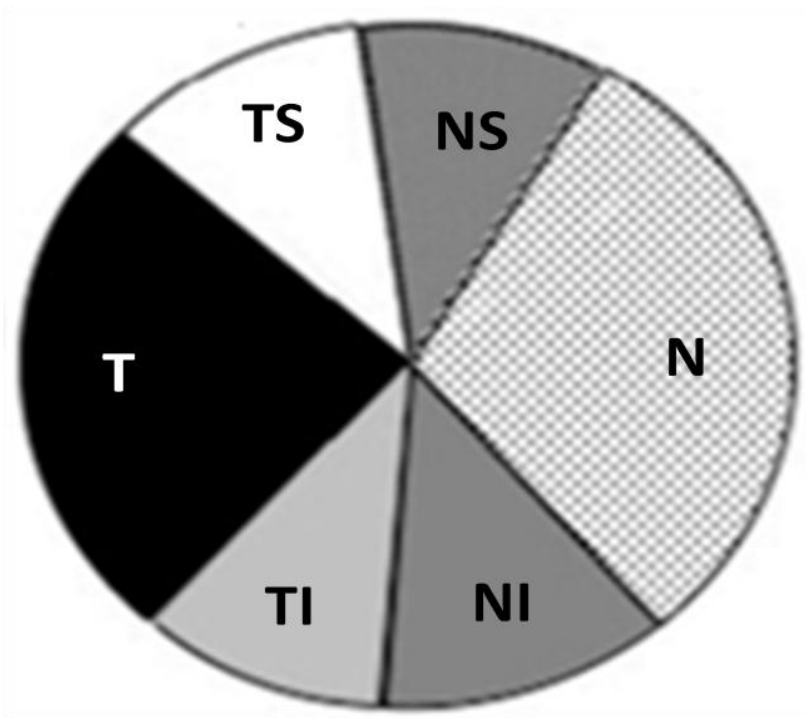




\subsubsection{Relações topográficas entre o posicionamento da fóvea e do nervo óptico}

A quantificação do ADF foi realizada pelo OCT-SD Spectralis. Um valor negativo indicou que a fóvea estaria localizada inferior ao DO, e um sinal positivo, que a fóvea estaria superior.

A DDF foi obtida por medição manual da linha desenhada entre a fóvea e o centro do DO no print out ou impresso da imagem da retina (IR 30 ART [HS]), usando a relação correspondente para o cálculo em micrômetros (Figura 7). Considerando que a linha média horizontal, a DDF e o DVF delimitam um triângulo retângulo (Figura 7), o DVF pode ser calculado aplicando a seguinte função trigonométrica [DVF = sen (ADF) DDF].

Figura 7 - llustração determinando os parâmetros usados na relação de posicionamento entre disco óptico e fóvea: 1. Ângulo disco-fóvea (ADF); 2. Diferença angular do defeito (DAD); 3. Distância disco-fóvea (DDF); 4. Desvio vertical da fóvea (DVF).

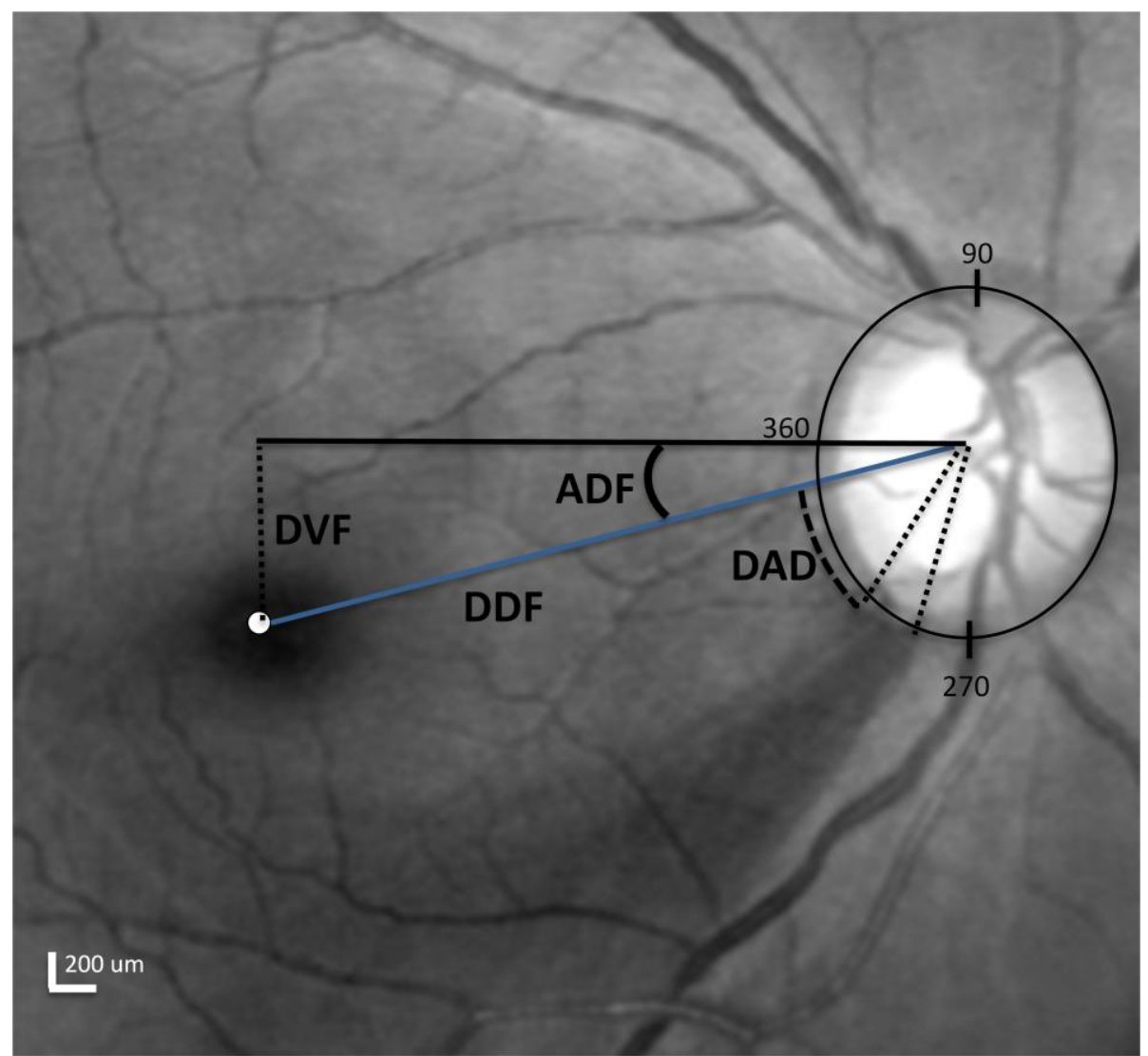


Os resultados dos pacientes do grupo DTI foram usados, ainda, para uma avaliação topográfica adicional, utilizando a distância angular do DTI e o eixo do alinhamento da fóvea para o disco. Esta variável foi nomeada como diferença angular do defeito (DAD) e leva em consideração a distância angular do limite superior do DTI subtraído do valor ADF para cada olho (Figura 7). Este limite superior do DTI foi determinado usando o valor de ângulo obtido no eixo $X$ do gráfico de espessura característico de dupla corcova TSNIT (na impressão do OCT), no qual a linha de medida da espessura da CFNR do paciente (linha preta) cruzava o ponto de significância para a área de espessura da CFNR, considerada como alterada (área vermelha) (Figura 8).

Figura 8 - Imagem demonstrando no gráfico TSNIT do OCT o local do inicio do defeito localizado. No caso, aproximadamente 300․ DAD será (360 - 300) - ADF

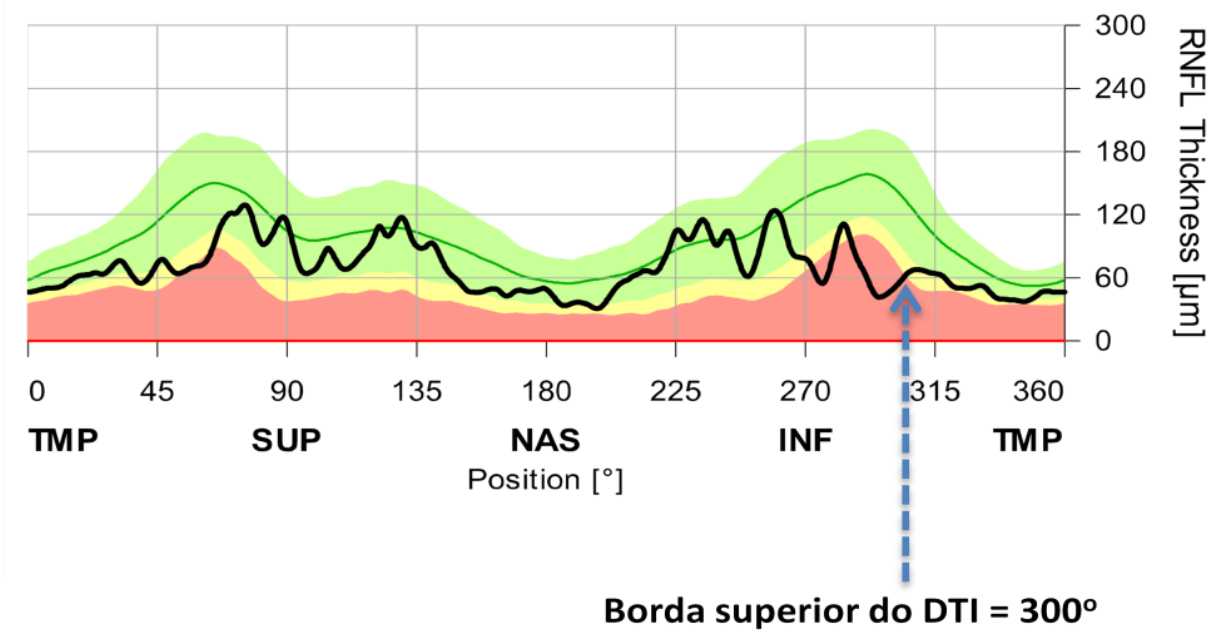

\subsection{Análise estatística}

Os dados foram analisados utilizando-se métodos de estatística descritiva e inferencial. Variáveis contínuas foram apresentadas como médias e desvio padrão (DP). A sensibilidade média da PPA e o número de pontos alterados nas regiões HS, BS, BN e SN foram normalizados pelo MD e pelo total de pontos alterados $(p<1 \%)$ no gráfico de TD, respectivamente, e foram considerados 
variáveis dependentes. Regressões univariadas com modelos de efeitos mistos e interceptos aleatórios foram utilizadas para se verificar o impacto do ADF, DVF e DAD em cada variável dependente. O método Benjamini-Hochberg foi aplicado para comparações múltiplas entre as diversas variáveis.

Valores de corte percentual selecionados (percentis 5\%, 10\%, 90\% e 95\%) de ADF, DVF e DAD, potencialmente capazes de determinar influências sobre defeitos da PPA foram investigados usando área sob a curva (AUC) de regressões binomiais para determinação de curvas ROC. Utilizou-se o programa Stata versão 14.2 (StataCorp LLC, College Station, TX, EUA) para as análises, e foram considerados resultados significativos quando $p<0,05 \%$. 
4. Resultados 


\subsection{Dados demográficos e clínicos}

Cinquenta e sete olhos de 35 pacientes com GPAA, com idade média de $59,7 \pm 23,3$ anos, foram incluídos neste estudo e divididos dois grupos: grupo DTI com 18 olhos, de 16 pacientes e grupo sem defeito localizado (controle) com 39 olhos, de 26 pacientes. Vinte participantes $(57,1 \%)$ eram mulheres e 15 homens $(42,9 \%)$. A amostra contou com 28 olhos direitos e 29 olhos esquerdos. Os

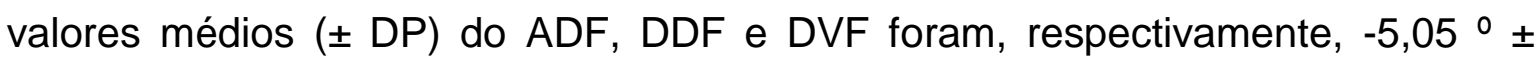
4,40으 $3976,7 \mu \mathrm{m} \pm 366,0 \mu \mathrm{m}$ e $-1346,6 \mu \mathrm{m} \pm 1609,0 \mu \mathrm{m}$. A média ( $\pm \mathrm{DP}$ ) de ADF no sexo feminino foi de $-4,85^{\circ} \pm 5,08^{\circ}$ e no sexo masculino, de $-5,68^{\circ} \pm 3,60^{\circ}$, sem diferença significativa $(p=0.4670)$.

Gráfico 1 - Comparação do ângulo disco-fóvea (ADF) e o número de olhos avaliados nos grupos DTI e controle, mostrando pouca disparidade entre os valores encontrados
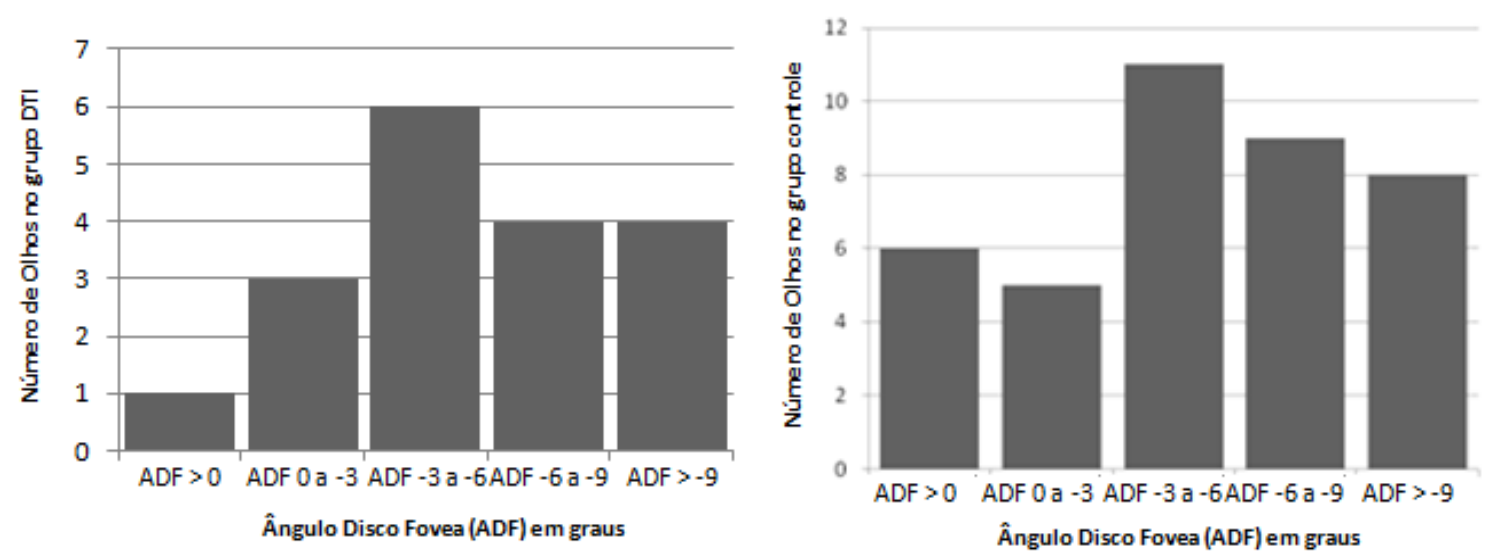

Os dados dos participantes, incluindo os resultados selecionados dos parâmetros dos $\mathrm{CV}$ e as variáveis topográficas de ambos os grupos estão apresentados na Tabela 2. Os valores de DAD do grupo sem DTI não foram medidos ou considerados para análise, uma vez que não houve contato evidente da linha TSNIT na área de espessura da CFNR alterada, pela ausência de defeito localizado detectável nesta região pelo OCT. 
Tabela 2 - Características demográficas e clínicas dos participantes demonstradas pela presença de DTI ou não, e de defeitos localizados na borda do nervo

\begin{tabular}{lccc}
\hline & DTI & Sem defeitos Localizados & P \\
\hline Número de olhos (pacientes) & $18(16)$ & $39(26)$ & -- \\
OD/OE & $10 / 8$ & $18 / 21$ & $0.58^{*}$ \\
PPA 10-2 (MD , dB) & $-5,4 \pm 6,3$ & $-4,9 \pm 5,1$ & $0.79^{* *}$ \\
Média CFNR, $\mu m$ & $74,9 \pm 11,2$ & $74,5 \pm 19,7$ & $0.92^{* *}$ \\
ADF, graus & $-5,7 \pm 4,5$ & $-4,9 \pm 4,5$ & $0.68^{* *}$ \\
DVF, $\mu$ m & $-1501,1 \pm 1154,9$ & $-1275,4 \pm 1809,3$ & $0.89^{* *}$ \\
DAD, graus & $43,3 \pm 17,3$ & $* * *$ & $* * *$ \\
\hline
\end{tabular}

TI: Infero-temporal; CV: Campo visual; CFNR: Camada de fibras nervosas da Retina; ADF: Ángulo discofóvea; DVF: Desvio vertical da fóvea; DAD: Diferença angular do defeito ângulo-disco; Variáveis contínuas presentes com média \pm desvio padrão (DP); *: Fisher's exact test; ** Mann-Whitney U test; ${ }^{* \star \star}$ não calculado.

\subsection{Correlação entre medidas da relação disco-fóvea com PPA programa 10-2}

Embora o ADF não tenha influenciado significativamente nenhum dos setores do PPA, programa 10-2, projetados no HS, as regressões univariadas mostraram correlações significativas de DVF e DAD com os setores em pacientes com DTI na rima do nervo óptico (Tabela 3); e apesar da sensibilidade do SN normalizado ter sido correlacionada com DVF no grupo sem defeito localizado $(p=0,006)$, nenhuma outra correlação significativa foi observada nesses pacientes. 
Tabela 3 - Resultados da correlação univariada entre fatores topográficos e perdas normalizadas em setores superiores selecionados da PPA, programa 10-2, em pacientes com DTI ou sem defeitos localizados

\begin{tabular}{|c|c|c|c|}
\hline Fator & $\begin{array}{c}\text { Normalizado } \\
\text { Variável dependente }\end{array}$ & $\begin{array}{l}\text { Sem defeito localizado } \\
\text { (P) }\end{array}$ & $\begin{array}{l}\text { DTI } \\
\text { (P) }\end{array}$ \\
\hline \multirow{8}{*}{$A D F$} & BS - sensibilidade & 0.933 & 0.124 \\
\hline & $B S-$ pontos & 0.672 & 0.438 \\
\hline & $B N$ - sensibilidade & 0.509 & 0.126 \\
\hline & $B N-$ pontos & 0.511 & 0.876 \\
\hline & HS - sensibilidade & 0.770 & 0.434 \\
\hline & $H S$ - pontos & 0.264 & 0.076 \\
\hline & SN - sensibilidade & 0.743 & 0.948 \\
\hline & $S N-$ pontos & 0.508 & 0.067 \\
\hline \multirow{8}{*}{$D V F$} & BS - sensibilidade & 0.090 & 0.559 \\
\hline & $B S-$ pontos & 0.293 & 0.114 \\
\hline & BN - sensibilidade & 0.435 & $<0.001$ \\
\hline & $B N-$ pontos & 0.387 & 0.004 \\
\hline & HS - sensibilidade & 0.349 & 0.528 \\
\hline & $H S$ - pontos & 0.971 & 0.019 \\
\hline & SN - sensibilidade & 0.006 & 0.027 \\
\hline & $S N-$ pontos & 0.522 & 0.002 \\
\hline \multirow{8}{*}{$D A D$} & $B S$ - sensibilidade & ${ }^{*}$ & $<0.001$ \\
\hline & $B S-$ pontos & * & $<0.001$ \\
\hline & $B N$ - sensibilidade & * & 0.468 \\
\hline & $B N-$ pontos & * & 0.001 \\
\hline & HS - sensibilidade & * & $<0.001$ \\
\hline & $H S$ - pontos & * & 0.002 \\
\hline & SN-sensibilidade & * & 0.027 \\
\hline & $S N-$ pontos & * & 0.012 \\
\hline
\end{tabular}

TI: Infero-temporal; BN: borda nasal; BS: borda superior; HS: hemicampo superior; SN: arco súpero-nasal; ADF: Ângulo Disco-Fóvea; DVF: Desvio Vertical da Fóvea; DAD: Diferença angular do defeito.

As comparações múltiplas demonstraram correlações fracas, mas significativas, entre todos os setores do PPA, DVF e DAD (valores de $R^{2}$ variando de 0,0016 a 0,1222 e de $p$ de 0,027 a 0,0001 nas diversas comparações) em pacientes com DTI (Figura 8 e Tabela 4). O grupo sem defeito localizado 
apresentou correlação significativa somente entre a sensibilidade SN e DVF $(p=0,006)$ (Tabela 4).

Tabela 4 - Resultados significativos de comparações múltiplas dos fatores topográficos que influenciaram as perdas normalizadas em setores superiores selecionados da PPA, programa 10-2, em pacientes com ou sem defeitos DTI

\begin{tabular}{|c|c|c|c|c|c|}
\hline $\begin{array}{c}\text { Variáveis Dependentes } \\
\text { Normalizadas }\end{array}$ & Fator & DTI & $\mathbf{P}$ & Posição & $\mathbf{R}^{2}$ \\
\hline$B N-$ sensibilidade & DVF & $\operatorname{sim}$ & 0.0001 & 1 & 0.0892 \\
\hline$B S$ - sensibilidade & DAD & $\operatorname{sim}$ & 0.0001 & 2 & 0.0283 \\
\hline$B S-$ pontos & DAD & $\operatorname{sim}$ & 0.0001 & 3 & 0.0062 \\
\hline HS - sensibilidade & DAD & $\operatorname{sim}$ & 0.0001 & 4 & 0.1178 \\
\hline$B N-$ pontos & DAD & $\operatorname{sim}$ & 0.001 & 5 & 0.1229 \\
\hline$S N-$ pontos & DVF & $\operatorname{sim}$ & 0.002 & 6 & 0.0172 \\
\hline$H S$ - pontos & DAD & $\operatorname{sim}$ & 0.002 & 7 & 0.0016 \\
\hline$B N-$ pontos & DVF & $\operatorname{sim}$ & 0.004 & 8 & 0.0139 \\
\hline SN- sensibilidade & DVF & não & 0.006 & 9 & 0.0681 \\
\hline$S N-$ pontos & DAD & $\operatorname{sim}$ & 0.012 & 10 & 0.0408 \\
\hline$H S-$ pontos & DVF & $\operatorname{sim}$ & 0.019 & 11 & 0.006 \\
\hline SN- sensibilidade & DVF & $\operatorname{sim}$ & 0.027 & 12 & 0.1055 \\
\hline SN- sensibilidade & DAD & $\operatorname{sim}$ & 0.027 & 13 & 0.0317 \\
\hline
\end{tabular}

TI: Temporal Inferior; BN: Borda nasal; BS: Borda superior; HS: Hemicampo superior; SN: Arco súperonasal; DVF: Desvio Vertical Foveal; DAD: Diferença angular do defeito.

Apenas valores de corte no percentil $95 \%$ de DVF (maior $-3264,5 \mu \mathrm{m}$ ) e a DAD (menor que $70,5^{\circ}$ ) foram considerados como limites discriminatórios, capazes de determinar significativamente as influências topográficas sobre a sensibilidade BS (para DVF: AUC $=0.60 ; 95 \% \mathrm{Cl}=0.50-0.71$; para DAD: $\mathrm{AUC}=$ $0,83$; IC $95 \%=0,70-0,912)$. Nenhum outro setor da PPA central mostrou ser afetado pelos outros valores de corte de DVF e DAD. 
Figura 9 - Imagem demonstrando exemplos de dois pacientes com ângulo disco-fóvea (ADF) comparáveis e defeitos localizados na borda temporal inferior apresentando diferentes padrões de perdas no setor de borda superior (BS) (delimitação cinza nos oito pontos superiores no desvio total ou total deviation) da PPA, programa 10-2. Note que o olho direito do segundo paciente apresenta pior perda de BS e desvio vertical da fóvea (DVF) grande e diferença angular de defeito (DAD) pequeno.

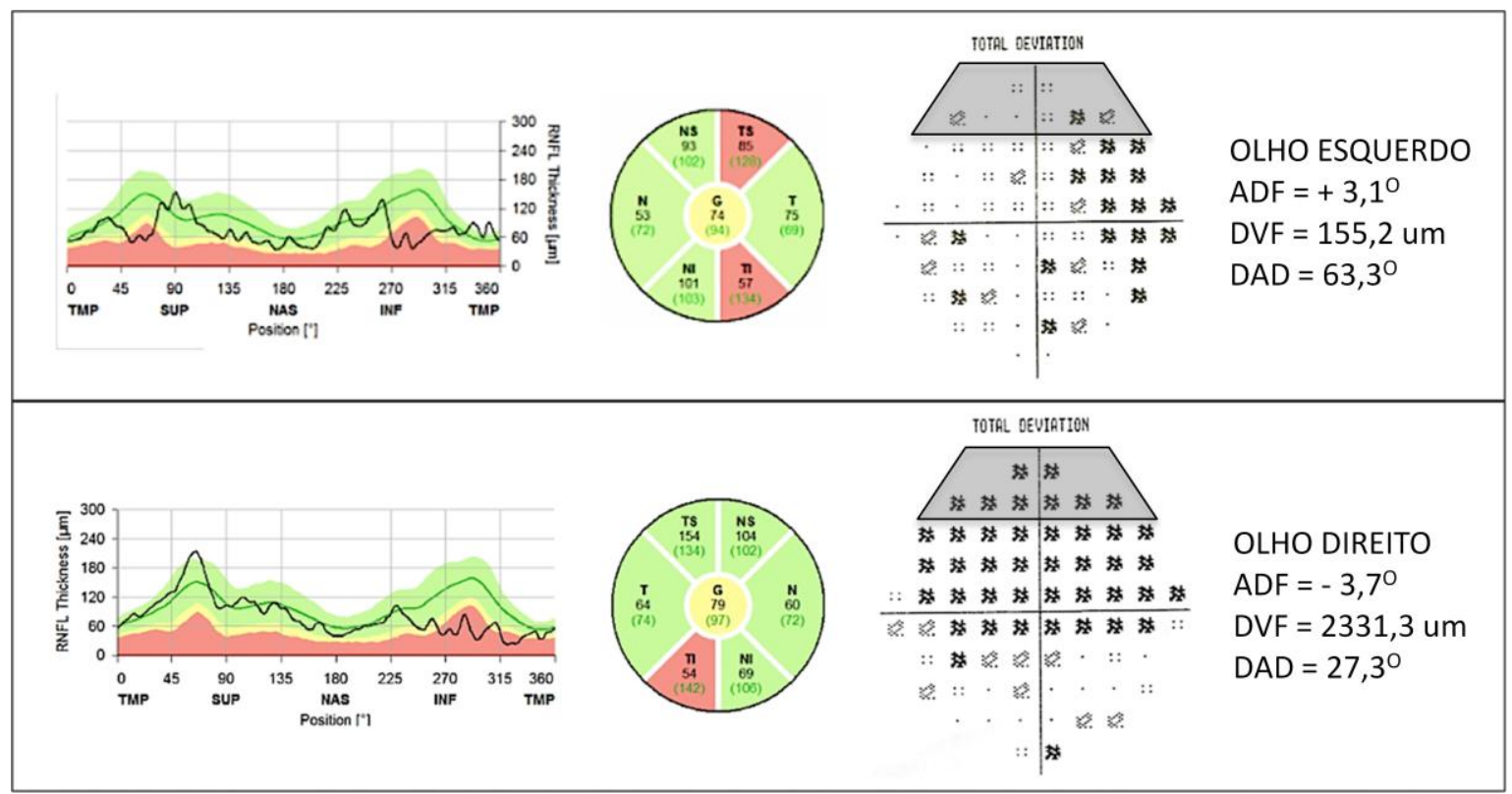


5. Discussão 
As funções visuais mais importantes para algumas atividades cotidianas, como leitura e identificação de objetos, dependem da integridade da fóvea e da região macular central. Pacientes com glaucoma em qualquer estágio podem apresentar lesão localizada na CFNR na região macular $(8,73,80)$. No entanto, o teste ainda mais utilizado para verificar o CV dos pacientes portadores de glaucoma usa a PPA, programa 24-2, subavaliando a região central $(55,90)$.

Existe ampla variação da espessura normal da CFNR, que sofre influência de idade, etnia, comprimento axial do olho, tamanho da CNO e sua posição relativa à fóvea. Este último tem sido considerado um dos fatores determinantes da distribuição da CFNR junto à rima da CNO (73). Esta variedade anatômica dos feixes, em conjunto com o ADF, poderia influenciar a correlação topográfica entre lesões da CFNR e perdas centrais de CV no glaucoma $(8,73,78-80)$.

Os padrões de desenvolvimento e progressão de defeitos nas fibras superior e inferior diferem em danos glaucomatosos. A rima neural e a espessura da CFNR nos olhos normais é, geralmente, mais ampla no quadrante inferior. Ademais, o CV superior, correspondente à CFNR inferior, está mais frequentemente envolvido nos estágios iniciais do glaucoma. Hood (19) sugeriu que, devido à característica da região temporal inferior da CNO com maior densidade da CFNR, a assimetria vertical de distribuição das fibras levaria à maior vulnerabilidade daquela região e ao dano glaucomatoso (denominada "zona de maior vulnerabilidade macular", ou MVZ). Alguns estudos mostram que a CFNR peripapilar inferior e as CGR na região nasal inferior da mácula seriam o melhor parâmetro para distinguir o nível de gravidade do glaucoma (66).

Como resultado da assimetria vertical da rima temporal, os olhos com ADF mais negativo, isto é, olhos em que o centro da fóvea está localizado mais inferiormente, podem apresentar perdas mais frequentes no HS em exames da PPA central, tais como o HFA 10-2 (Figura 10) $(73,89)$. 
Figura 10 - Imagem adaptada do modelo proposto por Hood et al. (2012) (90), estimando áreas de maior vulnerabiliade e sua influência na repercussão da lesão nas fibras maculares. A MVZ é a zona de vulnerabilidade macular na rima do disco óptico que se estende de $45^{\circ}$ a $69^{\circ}$.

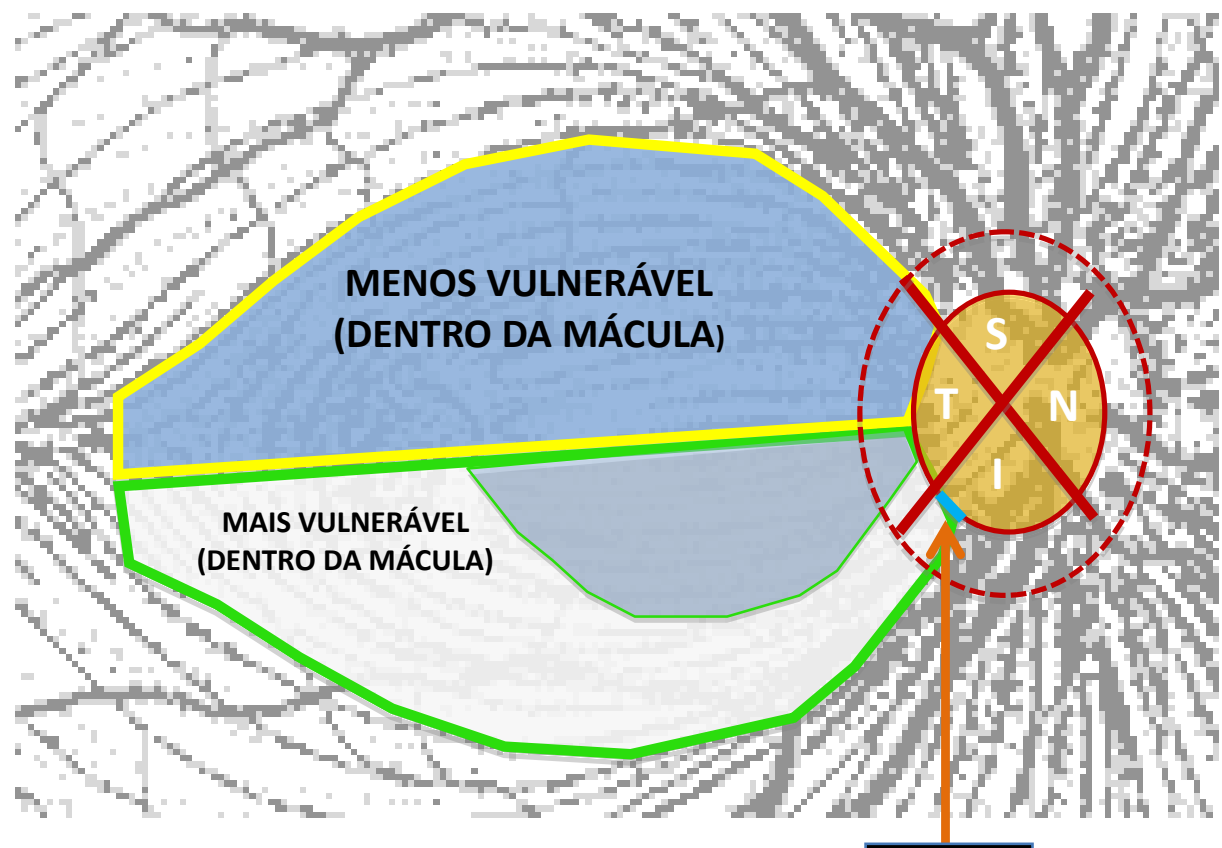

MVZ

Os valores médios do ADF encontrados no presente estudo foram -5,05 \pm $4,40^{\circ}$ ( $\left.\pm \mathrm{DP}\right)$, e estes valores assemelharam-se aos descritos na literatura (média de $-4^{\circ}$ a $\left.-7^{\circ}\right)(72,73,91)$. O grupo DTI apresentou maiores angulações negativas, quando comparado ao grupo sem defeito localizado, porém tal diferença não foi estatisticamente significativa, assim como outros fatores correlacionados (Tabela 1). Correlacionando o sexo dos participantes, o gênero masculino apresentou média de $\operatorname{ADF}$ de $-5,68^{\circ} \pm 3,6^{\circ}$ e o gênero feminino, de $-4,85^{\circ} \pm 5,0^{\circ}$, mas sem significância estatística. Estes resultados diferem dos de Amini et al. (81), que observaram que o gênero feminino apresentou mais valores negativos de ADF.

O grupo sem defeito localizado apresentou maiores valores $(2327.75 \mu \mathrm{m} \pm$ 1399.3 $\mu \mathrm{m})$ de DVF, comparado com o grupo DTI (2056.6 $\mu \mathrm{m} \pm 1218.7 \mu \mathrm{m})$, mas sem diferença significativa. Os valores referentes á média da DDF em ambos os grupos foi de $3,97 \mathrm{~mm}$, com leve diferença dos valores de $4.45 \mathrm{~mm}$ encontrados por Amini et al. (81). No entanto, Choi et al. (73) relataram que olhos com maiores DDF eram mais horizontalizados, ou seja, menores ADF. Fato que também foi 
observado quando comparados os valores de DDF versus ADF no conjunto de dados dos participantes deste estudo. Os valores dos MDs nas PPAs, no programa 10-2, foram mais negativos no grupo DTI $(-5.4 \mathrm{~dB} \pm 6.3 \mathrm{~dB})$, quando comparado com o grupo sem defeito localizado $(-4.9 \mathrm{~dB} \pm 5.1 \mathrm{~dB})$, mas sem significância estatística. Não houve diferença estatística na média da espessura da CFNR entre os dois grupos (74.9 $\pm 11.2 \mu \mathrm{m}$ no grupo com DTI e $74.5 \pm 19.7$ $\mu \mathrm{m}$ no grupo sem defeito localizado).

Ao se avaliarem os exames perimétricos dos pacientes com glaucoma apresentando DTI, com ADF diferentes, observou-se que as perdas no HS, bem como nos setores periféricos superiores e nasais das PPA, no programa 10-2 (BS, BN e SN), foram afetadas pelo posicionamento topográfico relativo entre a fóvea e o nervo óptico. Apesar de as medidas de ADF não se correlacionarem significativamente com nenhum dos setores das PPAs estudadas, os achados do presente estudo demonstraram que tanto o DVF quanto a DAD afetaram todos os setores, arbitrariamente definidos pelos autores, na PPA superior, no programa 10-2.

Choi et al. (73) e Hood et al. (78) mostraram que o ADF impacta a porção temporal da espessura da camada de fibras nervosas peripapilar e pode ser afetado pela DDF. Uma vez que o ADF e a DDF podem apresentar alguma correlação, foi proposto o DVF (que leva em consideração ambos os fatores) como um dos melhores parâmetros capazes de prever perdas no HS para o programa 10-2. De fato, o DVF maior foi significativamente associado à diminuição da sensibilidade média normalizada e ao número de pontos alterados em todos os setores periféricos definidos das PPAs no programa 10-2.

Uma vez que a influência do posicionamento anatômico da fóvea sobre os resultados observados no HS, programa 10-2, pode depender da distância relativa entre a fóvea (determinante centro da PPA) e o início do DTI (considerado como marco da perda central de CGR na região macular, mais próxima da fóvea), propôs-se a DAD como parâmetro alternativo para avaliação de perdas na PPA, programa 10-2, nas situações estudadas. Os valores de DAD menores foram significativamente correlacionados com diferenças médias de sensibilidade $e$ número de pontos alterados em todos os setores das PPAs estudadas. 
Especula-se que os resultados de correlação não significativos entre DVF e DAD com sensibilidade HS e BN, respectivamente, observados no grupo DTI, bem como a influência significativa do DVF na sensibilidade do SN no grupo sem defeito localizado podem ser resultado de uma alocação aleatória de pacientes de ambos os grupos com diferentes espessuras médias de CFNR na região temporal inferior.

Como esperado, tanto o DVF quanto a DAD apresentaram valores de corte capazes de prever de forma significativa as perdas nos pontos periféricos superiores da PPA, no programa 10-2, (sensibilidade BS média) de pacientes com DTI. De acordo com os achados deste estudo, pacientes que obtiveram valores de DVF maiores que $-3264.5 \mu \mathrm{m}$ e de DAD menores que $70,5^{\circ}$ podem apresentar desproporcionalmente pontos mais defeituosos nas posições superiores periféricas na PPA, programa 10-2, podendo estes valores serem usados na prática clínica como índice para maior risco de dano na PPA central.

Com base nos modelos de Hood para a relação estrutura-função, os DTI da CFNR produzem defeitos arqueados na PPA nas posições superiores $(73,89)$, áreas consideradas de maior vulnerabilidade. Considerando o impacto de defeitos nas zonas de vulnerabilidade na PPA central, bem como a observação de proporção moderada de pacientes com perdas na PPA, programa 10-2, antes das mudanças nas PPAs regulares no programa 24-2 (92), acredita-se que este estudo contribui com uma proposta de ajuste adicional entre as análises de OCT e PPA, de importância particular para pacientes com DTI, e risco de perdas visuais próximas à fixação central; ou mesmo, ainda, sem comprometimento detectável no programa 10-2 (Figura 11). 
Figura 11 - Imagem ilustrando variações da posição macular. Verificam-se os respectivos DAD, mostrando os ângulos 1,2 e 3. O ângulo 3, por ter menor valor, apresenta maior probabilidade de lesão na PPA central. (A, B, C) DVF das três posições. O DVF C, por ter maior valor, apresenta maior probabilidade de lesão na PPA central. A correlação foi positiva no estudo da DAD menor que 70,5o, e DVF com valor maior -3264.5 $\mu \mathrm{m}$.

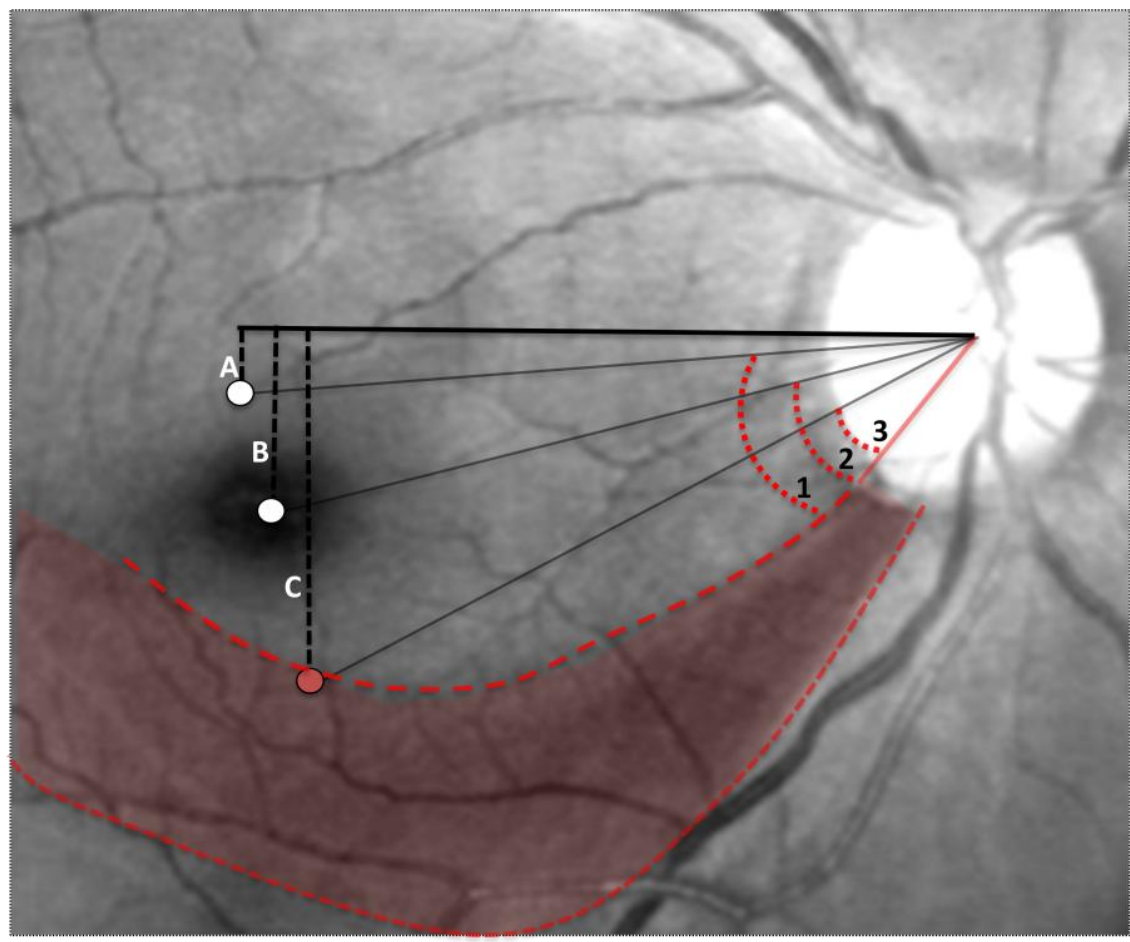

Algumas limitações podem ser consideradas para o presente estudo, a saber: amostra considerada relativamente pequena de olhos com DTI, uso de imagens de OCT e de medidas para o cálculo da DDF e da DAD, eventualmente influenciadas pela posição da cabeça durante o exame de OCT e PPA. Ressaltase, porém, que todas as medidas foram realizadas por um único examinador experiente (AGM), usando imagens de alta qualidade e ampliadas. Ainda, embora as medidas do ADF tomadas por OCT sejam, em geral, minimamente afetadas nos exames de rotina (82), foram tomados os cuidados para se estabilizar a posição da cabeça na testeira, mantendo alinhamento horizontal entre as pupilas dos pacientes, em posição primária do olhar.

Assim, melhor compreensão da variação topográfica da fóvea em relação à CNO, levando em conta as variações individuais, representa um avanço em 
termos de cuidado mais personalizado no glaucoma, respeitando, não só os detalhes próprios da doença, mas também as particularidades anatômicas de cada olho, face aos resultados funcionais obtidos. 
6. Conclusões 
Após análise dos resultados, concluiu-se que:

- Os valores do ADF, obtidos por meio de OCT, não mostraram correlações significativas com alterações de sensibilidade visual dos pontos superior da PPA central (programa 10-2) em pacientes com DTI e naqueles com GPAA sem DTI.

- O DVF, em relação à linha horizontal mediana e à distância angular entre o DTI e a fóvea (DAD), correlacionaram significativamente sobre a sensibilidade de pontos superiores na PPA, com programa 10-2, nos pacientes com DTI. 


\section{Referências Bibliográficas}


1. Almeida G, Cohen R. Glaucoma: história de uma doença. 1 edição. Rio de Janeiro: Cultura Médica; 2008.

2. Leffler $\mathrm{CT}$, et al. The early history of glaucoma: The glaucous eye (800 BC to 1050 AD). Clin Ophthalmol. 2015 Feb 2;9:207-15.

3. Quigley HA, Broman AT. The number of people with glaucoma worldwide in 2010 and 2020. Br J Ophthalmol. 2006 Mar;90(3):262-7.

4. Weinreb RN, Khaw PT. Primary open-angle glaucoma. Lancet. 2004 May;363(9422):1711-20.

5. Weinreb RN, Aung T, Medeiros FA. The Pathophysiology and Treatment of Glaucoma. JAMA. 2014 May;311(18):1901-11.

6. Mills RP, et al. Categorizing the stage of glaucoma from pre-diagnosis to endstage disease. Am J Ophthalmol. 2006 Jan;141(1):24-30.

7. Quigley HA. Number of people with glaucoma worldwide. $\mathrm{Br} \mathrm{J}$ Ophthalmol. 1996 May;80(5):389-93.

8. Tanabe $F$, et al. The interpretation of results of 10-2 visual fields should consider individual variability in the position of the optic disc and temporal raphe. Br J Ophthalmol. 2018 Mar;102(3):323-328.

9. Monteiro MLR. Avaliação da camada de fibras nervosas da retina nas afecções neuroftalmológicas da via óptica anterior. Rev Bras Oftalmol. 2012;71(2):125-38.

10. Perry VH, Oehler R, Cowey A. Retinal ganglion cells that project to the dorsal lateral geniculate nucleus in the macaque monkey. Neuroscience. 1984;12(4):1101-23.

11. Gupta N, Yücel YH. Brain changes in glaucoma. Eur J Ophthalmol. 2003;(13 Suppl. 3):S32-S35.

12. Yücel $\mathrm{YH}$, et al. Effects of retinal ganglion cell loss on magno-, parvo-, koniocellular pathways in the lateral geniculate nucleus and visual cortex in glaucoma. Prog Retin Eye Res. 2003 Jul;22(4):465-81.

13. Mittag TW, et al. Retinal damage after 3 to 4 months of elevated intraocular pressure in a rat glaucoma model. Investig Ophthalmol Vis Sci. 2000;41(11):3451-9.

14. Hare W, et al. Efficacy and safety of memantine, an NMDA-type open-channel blocker, for reduction of retinal injury associated with experimental glaucoma in rat and monkey. Surv Ophthalmol. 2001 May;(45 Suppl 3):S284-9; discussion S295-6. 
15. Haefliger $\mathrm{IO}$, et al. Potential role of nitric oxide and endothelin in the pathogenesis of glaucoma. Surv Ophthalmol. 1999 Jun;(43 Suppl 1):S51-8.

16. Tatton WG, Chalmers-Redman RME, Tatton NA. Apoptosis and anti-apoptosis signalling in glaucomatous retinopathy. Eur J Ophthalmol. $2001 \mathrm{Jul}$-Sep;(11 Suppl 2):S12-22.

17. Wang $\mathrm{N}$, et al. Protection of retinal ganglion cells against glaucomatous neuropathy by neurotrophin-producing, genetically modified neural progenitor cells in a rat model. Chin Med J (Engl). 2002;115(9):1394-400.

18. Bayer AU, et al. Association of glaucoma with neurodegenerative diseases with apoptotic cell death: Alzheimer's disease and Parkinson's disease. Am J Ophthalmol. 2002;133(1):135-7.

19. Hood DC. Improving our understanding, and detection, of glaucomatous damage: An approach based upon optical coherence tomography (OCT). Prog Retin Eye Res. 2017;57:46-75.

20. Marjanovic I. The Optic Nerve in Glaucoma. Belgrado: INTECH Open Access Publisher, 2011, p. 147-71.

21. Ramulu P. Glaucoma and disability: which tasks are affected, and at what stage of disease? Curr Opin Ophthalmol. 2009 Mar;20(2):92-8.

22. De Moraes CG V, et al. Risk factors for visual field progression in treated glaucoma. Arch Ophthalmol. 2011 May;129(5):562-8.

23. Susanna R, et al. Why Do people (Still) go blind from glaucoma? Transl Vis Sci Technol. 2015 Mar;4(2):1-12.

24. Susanna R, Vessani RM. Staging glaucoma patient: why and how? Open Ophthalmol J. 2009 Sep;3(2):59-64.

25. Chen PP. Blindness in patients with treated open-angle glaucoma. Ophthalmology. 2003 Apr;110(4):726-33.

26. Quigley HA. Identification of glaucoma-related visual field abnormality with the screening protocol of frequency doubling technology. Am J Ophthalmol. 1998 Jun;125(6):819-29.

27. Grant WM, Burke JF. Why do some people go blind from glaucoma? Ophthalmology. 1982 Sep;89(9):991-8.

28. Allingham RR, et al. Shields Textbook of Glaucoma, 6th Edition. Philadelphia: Lippincott Williams \& Wilkins, 2011. chap. 1, p. 3-23.

29. Francis BA, et al. Intraocular pressure, central corneal thickness, and 
prevalence of open-angle glaucoma: The Los Angeles Latino Eye Study. Am J Ophthalmol. 2008;146(5):741-6.

30. Gordon MO, et al. Validated prediction model for the development of primary open-angle glaucoma in individuals with ocular hypertension. Ophthalmology. 2007 Jan;114(1):10-9.

31. Varma R, et al. Prevalence of open-angle glaucoma and ocular hypertension in Latinos: The Los Angeles Latino Eye Study. Ophthalmology. 2004;111(8):1439-48.

32. Leske MC, et al. The Barbados Eye Study. Prevalence of open angle glaucoma. Arch Ophthalmol. 1994;112(6):821-9.

33. Rudnicka AR, et al. Variations in primary open-angle glaucoma prevalence by age, gender, and race: A Bayesian meta-analysis. Investig Ophthalmol Vis Sci. 2006;47(10):4254-61.

34. Friedman DS, et al. The prevalence of open-angle glaucoma among blacks and whites 73 years and older: The Salisbury Eye Evaluation glaucoma study. Arch Ophthalmol. 2006;124(11):1625-30.

35. Paranhos-Junior A, Omi CA, Prata-Junior J. III Consenso Brasileiro de Glaucoma Primário de Ângulo Aberto. São Paulo: Point B, editor, 2009.

36. Leske MC, et al. Factors for progression and glaucoma treatment: The Early Manifest Glaucoma Trial. Curr Opin Ophthalmol. 2004 Apr;15(2):102-6.

37. Medeiros FA, et al. Corneal thickness as a risk factor for visual field loss in patients with preperimetric glaucomatous optic neuropathy. Am J Ophthalmol. 2003;136(5):805-13.

38. Memarzadeh $F$, et al. Blood pressure, perfusion pressure, and open-angle glaucoma: The Los Angeles Latino eye study. Investig Ophthalmol Vis Sci. 2010;51(6):2872-7.

39. Foster PJ, et al. The definition and classification of glaucoma in prevalence surveys. Br J Ophthalmol. 2002;86(2):238-42.

40. Wolfs RCW, Bet al. Changing views on open-angle glaucoma: definitions and prevalences - The Rotterdam Study. Invest Ophthalmol Vis Sci. 2000;41(11):3309-21.

41. Boland MV, Quigley HA. Risk factors and open-angle glaucoma: classification and application. J Glaucoma. 2007;16(4):406-18.

42. Prum BE, et al. Primary open-angle glaucoma suspect preferred practice pattern ${ }^{\circledR}$ guidelines. Ophthalmology. 2016;123(1):P112-51. 
43. Smith ND, Glen FC, Crabb DP. Eye movements during visual search in patients with glaucoma. BMC Ophthalmol. 2012;12:45.

44. Thylefors B, Negrel AD. The global impact of glaucoma. Bull World Health Organ. 1994;72(3):323-6.

45. Resnikoff S, et al. Global data on visual impairment in the year 2002. Bull World Health Organ. 2004;82(11):844-51.

46. Pascolini D, Mariotti SP. Global estimates of visual impairment: $2010 . \mathrm{Br} \mathrm{J}$ Ophthalmol. 2012;96(5):614-8.

47. Bourne RRA, et al. Number of people blind or visually impaired by glaucoma worldwide and in world regions 1990 - 2010: a meta-analysis. PLoS One. 2016 Oct 20;11(10):e0162229.

48. Pascolini D, et al. 2002 global update of available data on visual impairment: a compilation of population-based prevalence studies. Ophthalmic Epidemiol. 2004 Apr;11(2):67-115.

49. McKean-Cowdin R, et al. Impact of Visual Field Loss on Health-Related Quality of Life in Glaucoma. Ophthalmology. 2008 Jun;115(6):941-8.e1.

50. Hattenhauer MG, J et al. The probability of blindness from open-angle glaucoma. Ophthalmology. 1998;105(11):2099-104.

51. Négrel A-D, Thylefors B. The global impact of eye injuries. Ophthalmic Epidemiol. 1998;5(3):143-69.

52. Hood DC, Raza AS. On improving the use of OCT imaging for detecting glaucomatous damage. Br J Ophthalmol. 2014 Jul;98 Suppl 2:ii1-9.

53. Mansoori T, Viswanath K, Balakrishna N. Quantification of retinal nerve fiber layer thickness in normal eyes, eyes with ocular hypertension, and glaucomatous eyes with SD-OCT. Ophthalmic Surg Lasers Imaging. 2010 Nov-Dec;41 Suppl:S50-7.

54. Heijl A, Lundqvist L. The frequency distribution of earliest glaucomatous visual field defects documented by automatic perimetry. Acta Ophthalmol. 1984;62(4):658-64.

55. Traynis I, et al. Prevalence and nature of early glaucomatous defects in the central $10^{\circ}$ of the visual field. JAMA Ophthalmol. 2014;132(3):291-7.

56. Schiefer $U$, et al. Increased detection rate of glaucomatous visual field damage with locally condensed grids: A comparison between fundus-oriented perimetry and conventional visual field examination. Arch Ophthalmol. 2003;121(4):458-65. 
57. Radius RL, Gonzales M. Anatomy of the lamina cribrosa in human eyes. Arch Ophthalmol. 1981;99(12):2159-62.

58. Boden C, Sample P a, Boehm AG, Vasile C, Akinepalli R, Weinreb RN. The structure-function relationship in eyes with glaucomatous visual field loss that crosses the horizontal meridian. Arch Ophthalmol. 2002;120(July 2002):90712.

59. Vrabec JP, Levin LA. The neurobiology of cell death in glaucoma. Eye. 2007;21:S11-4.

60. Wax MB, Tezel G. Neurobiology of glaucomatous optic neuropathy: diverse cellular events in neurodegeneration and neuroprotection. Mol Neurobiol. 2002;26(1):45-55.

61. Fujita K, et al. Reading performance in patients with central visual field disturbance due to glaucoma. Nihon Ganka Gakkai Zasshi. 2006;110(11):9148.

62. Coeckelbergh TRM, et al. The effect of visual field defects on driving performance: a driving simulator study. Arch Ophthalmol. 2002;120(11):150916.

63. Monsalve B, et al. Diagnostic ability of Humphrey perimetry, Octopus perimetry, and optical coherence tomography for glaucomatous optic neuropathy. Eye. 2017;31(3):443-51.

64. Hu R, et al. Comparison of standard automated perimetry, short-wavelength automated perimetry, and frequency-doubling technology perimetry to monitor glaucoma progression. Medicine (Baltimore). 2016 Feb;95(7):e2618.

65. van der Schoot J, et al. The ability of short-wavelength automated perimetry to predict conversion to glaucoma. Ophthalmology. 2010;117(1):30-4.

66. Bambo MP, et al. Evaluation of the macular ganglion cell-inner plexiform layer and the circumpapillary retinal nerve fiber layer in early to severe stages of glaucoma: correlation with central visual function and visual field indexes. Ophthalmic Res. 2017;57(4):216-23.

67. Rao HL, et al. Comparing glaucoma progression on 24-2 and 10-2 visual field examinations. PLoS One. 2015 May 15;10(5):e0127233.

68. Nevalainen $\mathrm{J}$, et al. Specification of progression in glaucomatous visual field loss, applying locally condensed stimulus arrangements. Graefe's Arch Clin Exp Ophthalmol. 2009;247(12):1659-69.

69. Park HY, et al. Visual field characteristics in normal-tension glaucoma patients with autonomic dysfunction and abnormal peripheral microcirculation. Am J 
Ophthalmol. 2012 Sep;154(3):466-475.e1.

70. Schiefer $U$, et al. Spatial pattern of glaucomatous visual field loss obtained with regionally condensed stimulus arrangements. Investig Ophthalmol Vis Sci. 2010;51(11):5685-9.

71. Bixenman WW, Von Noorden GK. Apparent foveal displacement in normal subjects and in cyclotropia. Ophthalmology. 1982;89(1):58-62.

72. Chauhan BC, Burgoyne CF. From clinical examination of the optic disc to clinical assessment of the optic nerve head: A paradigm change. Am $J$ Ophthalmol. 2013 Aug;156(2):218-227.e2.

73. Choi JA, et al. The foveal position relative to the optic disc and the retinal nerve fiber layer thickness profile in myopia. Investig Ophthalmol Vis Sci. 2014;55(3):1419-26.

74. De Silva DJ, et al. Optic disk size and optic disk-to-fovea distance in preterm and full-term infants. Investig Ophthalmol Vis Sci. 2006;47(11):4683-6.

75. Knaapi $L$, et al. Determining the size of retinal features in prematurely born children by fundus photography. Acta Ophthalmol. 2015;93(4):339-41.

76. Jonas RA, et al. Optic disc - fovea distance, axial length and parapapillary zones. The Beijing Eye Study 2011. PLoS One. 2015 Sep 21;10(9):e0138701.

77. Kimura $Y$, et al. Retinal nerve fiber layer defects in highly myopic eyes with early glaucoma. Invest Ophthalmol Vis Sci. 2012;53(10):6472-8.

78. Hood DC, et al. Glaucomatous damage of the macula. Prog Retin Eye Res. 2013 Jan;32:1-21.

79. Hwang $\mathrm{YH}$, et al. Macular ganglion cell analysis for early detection of glaucoma. Ophthalmology. 2014;121(8):1508-15.

80. Ohkubo S, et al. Focal relationship between structure and function within the central 10 degrees in glaucoma. Investig Ophthalmol Vis Sci. 2014;55(8):5269-77.

81. Amini N, et al. Influence of the disc - fovea angle on limits of RNFL variability and glaucoma discrimination. Invest Ophthalmol Vis Sci. 2014 Oct 9;55(11):7332-42.

82. Denniss J, Turpin A, McKendrick AM. Individualized structure-function mapping for glaucoma: Practical constraints on map resolution for clinical and research applications. Investig Ophthalmol Vis Sci. 2014;55(3):1985-93.

83. Hood DC, et al. Early glaucoma involves both deep local , and shallow 
widespread, retinal nerve fiber damage of the macular region. Invest Ophthalmol Vis Sci. 2014 Feb 3;55(2):632-49.

84. Mills RP, et al. Categorizing the stage of glaucoma from pre-diagnosis to endstage disease. Am J Ophthalmol. 2006 Jan;141(1):24-30.

85. Schimiti RB, et al. Full-threshold versus Swedish Interactive Threshold Algorithm (SITA) in normal individuals undergoing automated perimetry for the first time. Ophthalmology. 2002 Nov;109(11):2084-92; discussion 2092.

86. Jampel HD, et al. Assessment of Visual Function in Glaucoma. Ophthalmology. 2011 May;118(5):986-1002.

87. Glen FC, et al. Do patients with glaucoma have difficulty recognizing faces? Investig Ophthalmol Vis Sci. 2012 Jun;53(7):3629-37.

88. Lin J-C, Yang M-C. Correlation of visual function with health-related quality of life in glaucoma patients. J Eval Clin Pract. 2010 Feb;16(1):134-40.

89. Garway-Heath DF, et al. Mapping the visual field to the optic disc in normal tension glaucoma eyes. Ophthalmology. 2000;107(10):1809-15.

90. Hood DC, et al. The nature of macular damage in glaucoma as revealed by averaging optical coherence tomography data. Transl Vis Sci Technol. 2012;1(1):3.

91. He L, et al. Anatomic vs. Acquired image frame discordance in spectral domain optical coherence tomography minimum rim measurements. PLoS One. 2014 Mar 18;9(3):e92225.

92. De Moraes CG, et al. 24-2 visual fields miss central defects shown on 10-2 tests in glaucoma suspects, ocular hypertensives, and early glaucoma. Ophthalmology. 2017;124(10):1449-56. 
8. Anexos 


\section{ANEXO A}

\section{COMPROVANTE DE APROVAÇÃO DO COMITÊ DE ÉTICA}

\begin{tabular}{cc|}
\hline USP - FACULDADE DE \\
MEDICINA DE RIBEIRÃO \\
PSIBEIRẮ \\
PRETO DA USP
\end{tabular}

\section{PARECER CONSUBSTANCIADO DO CEP}

\section{DADOS DO PROJETO DE PESQUISA}

TItulo da Pøsquisa: CORRELAÇÅO DE VARIAVEIS ANATOMO-FUNCIONAIS EM PACIENTES COM NEUROPATIA OPTICA GLAUCOMATOSA

Pesquisador: ALEXIS GALENO MATOS

Área Tematica:

Versao: 3

CA.AE: 58299516.7 .0000 .5440

Instıtulçăo Proponente: HOSPITAL DAS CLINICAS DA FACULDADE DE MEDICINA DE RPUSP

Patrocinador Princlpal: FInanclamento Proprio

DADOS DO PARECER

Número do Parecer: 1.887.298

\section{Apresentaçaso do Projeto:}

O glaucoma e uma doença dos olhos que em geral ocorre por aumento da pressăo do olho (pressáo Intraocular). Quando năo tratada, esta pressăo aumentada afeta a visăo ate causar ceguelra. Segundo trabalhos da Organizaçăo Mundlal da Saude (OMS), o glaucoma e responsavel pela segunda malor causa de cegueira no mundo, tendo grande repercussăo socloeconomica.A doença leva a morte prematura de celulas do fundo do olho que formam a retina e nervo optico e assim afeta a visalo progresslvamente ate a perda total da capacidade de enxergar. E uma doença que normalmente năo apresenta sintomas. Essa pesquisa se justifica pela possiblildade de se alcançar um malor conhecimento sobre a doença e como ela se apresenta em varios exames oftalmologlcos nas fases Iniclals, buscando melos de reallzar um dlagnostico mals precoce, levando em consideraçăo as varlaçర్es da anatomla do fundo do olho. Sera reallzada realizando varlos exames oftalmologlcos em paclentes glaucomatosos e sadlos tentando correlacionar com as varlaçbes anatomicas Individuals, como por exemplo, a distancla e 0 angulo entre a macula e o nervo optico.

Objetivo da Pesquisa:

A pesquisa pretende anallsar a variabilldade anatomica de posiclonamento relativo da cabeça do disco optico, correlacionando achados estruturals e funclonals locallzados em paclentes com 


\begin{tabular}{|c|c|}
\hline$S P \cdot R I B E I R$ & $\begin{array}{l}\text { USP - FACULDADE DE } \\
\text { MEDICINA DE RIBEIRÃO } \\
\text { PRETO DA USP }\end{array}$ \\
\hline
\end{tabular}

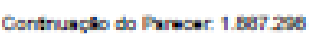

glaucoma, visando a (a) correlaclonar os valores do angulo dlsco-fovea e a distancla disco-fovea, medidas com OCT de nervo optico e macula, com o Indlce de campo visual central (CFI) e numero de pontos alterados no teste perimetrico 10.2 em paclentes com GPAA e GPN com "notch" e/ou Hoyt temporals Inferiores, (b) correlacionar achados microestruturals na rima neural temporal inferior do dlsco optico desses pacientes, por melo dos exames AO, OCT e HRT III, com alteraçßోes nos testes perimetricos 10.2 e 24.2, e (c) anallsar diferenças na evoluçăo de lesరీes glaucomatosas locallzadas e generallzadas, do ponto de vista estrutural (OCT, HRT III, AO, retinografla de polo posterior), correlaclonado-as com alteraçăo eletronslologicas e funcionals oculares, entre paclentes com neuropatla glaucomatosa e sujeltos normals.

\section{Avallaçă dos Rlacos $\theta$ Benefliclos:}

Riscos: todas as pesqu/sas apresentam riscos; quanto malores e mals evidentes, malores serd̆o os culdados para minimiza-los e proteger 05 particlpantes. Serăo admisslvels as pesquisas com seres humanos quando o risco justifica os beneficlos. Neste caso, o pesquisador expllichtou os riscos e o compromisso com o sigllo $e$ a confidenclalldade.

\section{Comentarlos e Consideraçסes sobre a Pesqulsa:}

A coleta de dados da pesquisa sera felta no Setor de Glaucoma do HCFMRP, com paclentes de ambos os sexos, com Idade entre 18 e 80 anos, que serăo divididos em tres grupos, conforme o criterios de dlagnostico (grupo 1, controle: sujeltos sem doenças oculares ou afeç̧bes slstemlcas que repercutam com alteraçbes oculares; grupo 2: paclentes com dlagnostico de glaucoma primario de angulo aberto (GPAA), Inclulndo os dlagnosticas como de "pressáo normal", que apresentem notch e hoyt locallzados na regláo temporal Inferior, Julgados por dols oftalmologistas especlallstas em glaucoma por melo de Imagens retinograncas e OCT; grupo 3: paclentes com dlagnostico de glaucoma, inciulndo outras formas da doença, sem lesळes avançadas ou locallzadas), com 50 Indlviduos, ap0s assinarem o TCLE. Foram expllcitados os criterios de Inclusăo e de exclusăo.

Consıderaçסөs sobre 0 Termos de apresentaçà obrigatorla:

Todos 05 documentos necessarios foram apresentados.

\section{Recomendaç0日s:}

O pesqulsador atendeu as recomendaçbes sollcitadas no parecer anterior. 


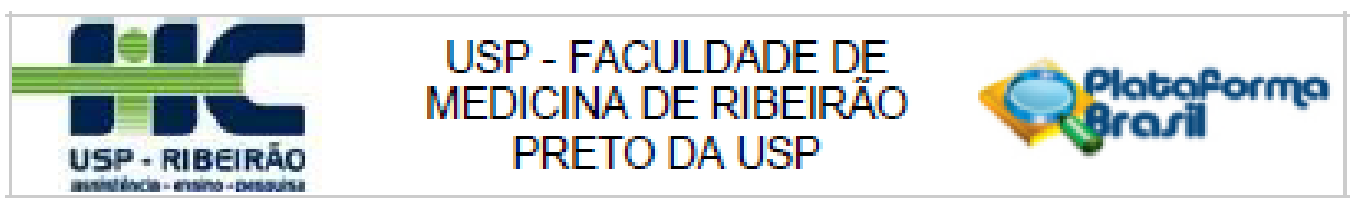

Contruaçso do Marweer. 1. Bat 296

\begin{tabular}{|c|c|c|c|c|}
\hline Orçamento & ORCAMENTO.dOCX & $19: 03: 25$ & MATOS & Acelto \\
\hline $\begin{array}{l}\text { Projeto Detalhado / } \\
\text { Brochura } \\
\text { Investigador }\end{array}$ & PROJETO.dOCX & $\begin{array}{c}02 / 08 / 2016 \\
19: 03: 03\end{array}$ & $\begin{array}{l}\text { ALEXIS GALENO } \\
\text { MATOS }\end{array}$ & Acelto \\
\hline $\begin{array}{l}\text { TCLE / Termos de } \\
\text { Assentimento / } \\
\text { Justificativa de } \\
\text { Ausencla }\end{array}$ & TCLE.pd' & $\begin{array}{c}04 / 04 / 2016 \\
21: 56: 07\end{array}$ & $\begin{array}{l}\text { ALEXIS GALENO } \\
\text { MATOS }\end{array}$ & Acelto \\
\hline $\begin{array}{l}\text { Declaraçăo de } \\
\text { Insttulçăo e } \\
\text { Infraestrutura }\end{array}$ & ProjetoassinadoCEP.pdf & $\begin{array}{c}04 / 04 / 2016 \\
21: 48: 13\end{array}$ & $\begin{array}{l}\text { ALEXIS GALENO } \\
\text { MATOS }\end{array}$ & Acelto \\
\hline Folna de Rosto & follharosto.pdf & $\begin{array}{c}04 / 04 / 2016 \\
21: 46: 32\end{array}$ & $\begin{array}{l}\text { ALEXIS GALENO } \\
\text { MATOS } \\
\end{array}$ & Acelto \\
\hline
\end{tabular}

situaçăo do Parecer:

Aprovado

Necesalta Apreclaçåo da CONEP:

Năo

RIBEIRAO PRETO, 07 de Dezembro de 2016

A.ssinado por:

MARCIA GUIMARAES VILLANOVA

(Coordenador) 


\section{ANEXO B}

\section{TERMO DE CONSENTIMENTO LIVRE E ESCLARECIDO}

Você está sendo convidado(a) como voluntário(a) a participar da pesquisa:

Estudo da variabilidade anatômica da camada de fibras nervosas da retina em pacientes com neuropatia óptica

Pesquisadores Colaboradores:

Prof. Dr. Jayter Silva de Paula - Contato: (16) 3602-2528

Dr. Marcelo Jordão L. da Silva - Contato: (16) 3602-2499

Dr. Alexis Galeno Matos - Contato: (16) 3602-2323

Após ser esclarecido(a) sobre as informações a seguir, no caso de aceitar fazer parte do estudo, assine ao final deste documento, que está em duas vias. Uma delas é sua e a outra do pesquisador responsável.

\section{Explicação da Justificativa e Objetivos do Estudo}

O glaucoma é uma doença dos olhos que em geral se ocorre por aumento da pressão do olho (pressão intraocular). Quando não tratada, esta pressão aumentada afeta a visão até causar cegueira.

Segundo a Organização Mundial da Saude (OMS) é responsável pela segunda maior causa de cegueira no mundo, tendo grande repercussão socioeconomica.

A doença leva a morte prematura de células do fundo do olho que formam a retina e nervo óptico e assim afeta a visão progressivamente até a perda total da capacidade de enxergar. É uma doença que normalmente não apresenta sintomas.

Essa pesquisa se justifica pela oportunidade de um maior conhecimento sobre a doença e como ela se apresenta precocemente em vários exames oftalmológicos, buscando meios de realizar um diagnóstico mais precoce, levando em consideração as variações anatômicas.

\section{Explicando a participação no estudo}

Sua participação é voluntária. Não haverá compensação econômica ou financeira por sua participação nesse estudo, nem haverá previsão de nenhum ressarcimento de gastos referentes a transporte, alimentação e demais custos com despesas relacionados à sua participação, mas também você não terá gastos com os procedimentos, exames e consultas médicas.

Durante o estudo você deverá comparecer às visitas de acompanhamento, conforme instruído pelo seu médico. Você poderá informar seu médico caso venha a mudar qualquer medicação em uso.

Você pode abandonar o estudo quando quiser. Se decidir abandonar sua participação nesse estudo, isso não trará nenhuma consequência no tratamento que está fazendo com a equipe médica nesta instituição. 


\section{Explicando quanto a prováveis benefícios}

Este estudo é direcionado a pacientes portadores de glaucoma em tratamento adequado e visa realizar exames oftalmológicos variados buscando avaliar as possíveis alterações e correlacionar com as variações anatômicas individuais, desenvolvendo uma análise mais individualizada de acordo com as características especificas de cada paciente.

Procura-se desta forma conhecer e indicar o exame mais específico de acordo com o aspecto anatômico para um diagnóstico mais precoce e um acompanhamento com exames mais específicos.

Procedimentos: $O$ que acontecerá se eu decidir participar?

Você deverá ler com calma este documento e ter todas suas dúvidas respondidas pelos pesquisadores, podendo assinar esse termo de consentimento livre e esclarecido apenas se concordar em participar deste estudo. Uma cópia será entregue a você e você deverá responder algumas perguntas sobre os medicamentos que utiliza atualmente e sobre os medicamentos que você já utilizou para tratar o glaucoma. Além disso, o médico irá questioná-lo sobre sua saúde e doenças que já foram tratadas. De posse destas respostas e após um exame clínico oftalmológico, o médico irá informar se você pode ou não pode participar do estudo. Assim que o médico autorizar a sua participação no estudo, ele irá realizar alguns exames em seus olhos.

Os procedimentos de exame ocorrerão no Hospital das Clínicas de Ribeirão Preto da Faculdade de Medicina de Ribeirão Preto, no campus da USP, Ribeirão Preto, São Paulo.

\section{- Explicando o acompanhamento e os exames oftalmológicos}

Todos os pacientes serão submetidos aos exames que seguem, todos não invasivos, não dolorosos e sem risco para sua saúde ocular.

O exame oftalmológico constará de: medida da acuidade visual, avaliação das alterações biomicroscopicas, medida da pressão intra-ocular e avaliação do nervo óptico.

Exames computadorizados: campo visual, além de exames de imagem do polo posterior do olho como a retinografia, tomografia de coerência óptica, adaptive optics e outro exames de eletrofisiologia como a eletrorrenografia e o potencial visual evocado.

Veja a seguir a explicação de como eles são realizados, esclarecendo que deverão ser realizados neste mesmo hospital.

Acuidade Visual: é uma medida da visão feita através da leitura de letras em uma tabela a uma determinada distância;

Biomicroscopia: é um exame da parte da frente dos olhos, com um aparelho composto por luzes e lentes;

Tonometria: é a medida da pressão dos olhos, feita com um pequeno aparelho, após a administração de 1 gota de anestésico tópico em cada olho;

Fundoscopia: é o exame da retina (parte interna do fundo do olho), feito através de uma luz e uma lente;

Perimetria ou Campo Visual: é um exame que testa a visão através da apresentação de vários pontos luminosos dentro de uma cúpula, no qual o paciente aciona um botão do aparelho quando enxerga tais pontos. 
Retinografia: é um exame dado por fotografias do fundo do olho após a aplicação de colírio para dilatar a pupila.

Tomografia de coerência óptica, HRTIII, Adaptive Optics: são exames obtidos por imagens do fundo do olho, não precisando obrigatoriamente a dilatação da pupila.

Eletrorretinograma e potencial visual evocado: testes eletrofisiológicos que visam avaliar a resposta da retina por meio de sensores colocados no paciente ao observar estímulos em uma tela.

- Liberdade de Recusa e Garantia de Esclarecimentos e Sigilo

Você será esclarecido(a) sobre a pesquisa em qualquer aspecto que desejar.

Você é livre para recusar-se a participar, retirar seu consentimento ou interromper a participação a qualquer momento. A sua participação é voluntária (não sendo obrigado a participar) e a recusa em participar não irá acarretar qualquer penalidade ou perda de benefícios, nem mesmo mudança no seu seguimento ou tratamento neste serviço.

Os pesquisadores irão tratar a sua identidade com padrões profissionais de sigilo. Os resultados serão demonstrados a você e permanecerão confidenciais. Seu nome ou o material que indique a sua participação não será liberado sem a sua permissão. Você não será identificado(a) em nenhuma publicação, texto, aula ou apresentação teórica que possa resultar deste estudo. Uma cópia deste consentimento informado será arquivada na Divisão de Oftalmologia (setor de glaucoma) do Hospital das Clínicas de Ribeirão Preto e outra será fornecida a você.

A sua participação nesta pesquisa é VOLUNTÁRIA e seu nome vai ficar em segredo (através de codificação numérica) e anonimato; não aparecerá nos resultados da pesquisa, não havendo, assim, possibilidade de ser identificado (mantendo total privacidade). Além disso, se for de sua vontade, nos comprometemos a prestar informações ou esclarecimentos atualizados durante todas as fases do estudo (inclusive dos seus resultados pessoais), mesmo que esses possam afetar a sua vontade de continuar participando. Caso concorde em participar, poderá também desistir em qualquer momento, sem qualquer prejuízo para o seu tratamento ou para o acompanhamento clínico durante e após a conclusão do trabalho.

Por acaso, se no decorrer do estudo for identificada alguma alteração nos exames oftalmológicos e/ou nos testes laboratoriais que forem feitos eu:

( ) Gostaria de ser comunicado

( ) Não gostaria de ser comunicado

por um dos médicos responsáveis pelo projeto e que poderá me orientar sobre o significado dessas alterações e de quais providências deverão ser tomadas caso haja necessidade de tratamento, além de me encaminhar se necessário para um especialista e também prestar esclarecimentos sobre os possíveis riscos das alterações.

Nome do participante:

assinatura: data:

Nome do pesquisador:

assinatura: data: 